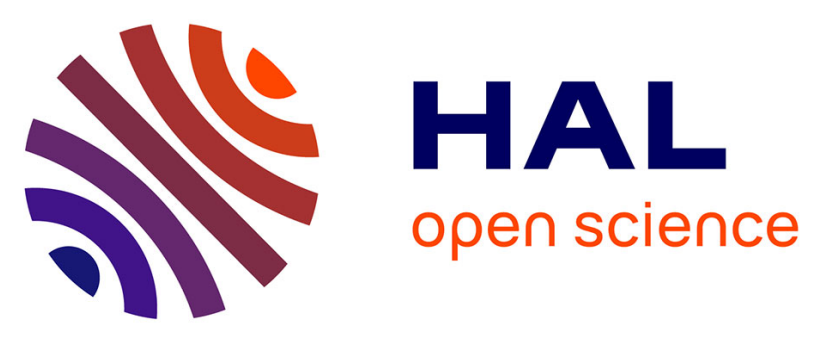

\title{
Efficient incorporation and protection of lansoprazole in cyclodextrin metal-organic frameworks
}

\author{
Xue Li, Marianna Porcino, Charlotte Martineau-Corcos, Tao Guo, Ting \\ Xiong, Weifeng Zhu, Gilles Patriarche, Christine Péchoux, Barbara Perronne, \\ Alia Hassan, et al.
}

\section{To cite this version:}

Xue Li, Marianna Porcino, Charlotte Martineau-Corcos, Tao Guo, Ting Xiong, et al.. Efficient incorporation and protection of lansoprazole in cyclodextrin metal-organic frameworks. International Journal of Pharmaceutics, 2020, 585, 10.1016/j.ijpharm.2020.119442 . hal-02844079

\author{
HAL Id: hal-02844079 \\ https://hal.science/hal-02844079
}

Submitted on 12 Nov 2020

HAL is a multi-disciplinary open access archive for the deposit and dissemination of scientific research documents, whether they are published or not. The documents may come from teaching and research institutions in France or abroad, or from public or private research centers.
L'archive ouverte pluridisciplinaire $\mathbf{H A L}$, est destinée au dépôt et à la diffusion de documents scientifiques de niveau recherche, publiés ou non, émanant des établissements d'enseignement et de recherche français ou étrangers, des laboratoires publics ou privés.

\section{(ㅇ)(1) $\$$}

Distributed under a Creative Commons Attribution - NonCommercial - NoDerivatives $\mid 4.0$ 


\title{
Efficient incorporation and protection of lansoprazole in cyclodextrin metal-organic frameworks
}

\author{
Xue Li $^{\mathrm{a}}$, Marianna Porcino ${ }^{\mathrm{b}}$, Charlotte Martineau-Corcos ${ }^{\mathrm{c}, \mathrm{d}}$, Tao Guo ${ }^{\mathrm{e}}$, Ting Xiong ${ }^{\mathrm{f}}$, Weifeng Zhu ${ }^{\mathrm{f}}$, \\ Gilles Patriarche $^{\mathrm{g}}$, Christine Péchoux ${ }^{\mathrm{h}}$, Barbara Perronne ${ }^{\mathrm{i}}$, Alia Hassan ${ }^{\mathrm{i}}$, Rainer Kümmerle ${ }^{\mathrm{i}}$, \\ Alexandre Michelet $^{\mathrm{j}}$, Anne Zehnacker-Rentien ${ }^{\mathrm{a}}$, Jiwen Zhang ${ }^{\mathrm{e}, \mathrm{f}}$, Ruxandra Gref ${ }^{\mathrm{a}, *}$ \\ a Université Paris-Saclay, CNRS, Institut des Sciences Moléculaires d'Orsay, 91405 Orsay, France \\ ${ }^{\mathrm{b}}$ Université d'Orléans, CEMHTI UPR CNRS 3079, F-45071 Orléans, France \\ c Université Paris Saclay, ILV UMR CNRS 8180, Université de Versailles St-Quentin en Yvelines, 78035 Versailles, France \\ ${ }^{\mathrm{d}}$ Institut Universitaire de France (IUF), 75005 Paris, France \\ e Center for Drug Delivery Systems, Shanghai Institute of Materia Medica, Chinese Academy of Sciences, 201210 Shanghai, China \\ ${ }^{\mathrm{f}}$ Key Laboratory of Modern Preparation of TCM, Ministry of Education, Jiangxi University of Traditional Chinese Medicine, 330004 Nanchang, China \\ g Université Paris-Saclay, CNRS, Centre de Nanosciences et de Nanotechnologies, 91120 Palaiseau, France \\ ${ }^{\mathrm{h}}$ Université Paris-Saclay, INRAE, AgroParisTech, GABI, 78350 Jouy-en-Josas, France

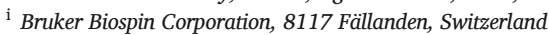 \\ j PerkinElmer, 16 avenue du Québec, 91140 Villebon sur Yvette, France
}

\section{A R T I C L E I N F O}

Keywords

Cyclodextrin

Metal-organic frameworks

Lansoprazole

Inclusion complex

Drug stability

\begin{abstract}
A B S T R A C T
Lansoprazole (LPZ) is an acid pump inhibitor, which readily degrades upon acidic or basic conditions and under heating. We investigated here LPZ stability upon incorporation in particles made of cyclodextrin metal-organic frameworks (CD-MOFs). LPZ loaded CD-MOFs were successfully synthesized, reaching high LPZ payloads of $23.2 \pm 2.1 \mathrm{wt} \%$, which correspond to a molar ratio of 1:1 between LPZ and $\gamma$-CD. The homogeneity of LPZ loaded CD-MOFs in terms of component distribution was confirmed by elemental mapping by STEM-EDX. Both $\mathrm{CTAB}$, the surfactant used in the CD-MOFs synthesis, and LPZ compete for their inclusion in the CD cavities. $\mathrm{CTAB}$ allowed obtaining regular cubic particles of around $5 \mu \mathrm{m}$ with $15 \mathrm{wt} \%$ residual CTAB amounts. When LPZ was incorporated, the residual CTAB amount was less than $0.1 \mathrm{wt} \%$, suggesting a higher affinity of LPZ for the CDs than CTAB. These findings were confirmed by molecular simulations. Vibrational circular dichroism studies confirmed the LPZ incorporation inside the CDs. Solid-state NMR showed that LPZ was located in the CDs and that it remained intact even after three years storage. Remarkably, the CD-MOFs matrix protected the drug upon thermal decomposition. This study highlights the interest of CD-MOFs for the incorporation and protection of LPZ.
\end{abstract}

\section{Introduction}

Cyclodextrin-based metal-organic frameworks (CD-MOFs) have emerged as one of the latest classes of crystalized porous MOFs particles. Since their discovery in 2010 (Smaldone et al., 2010), CD-MOFs have attracted growing interest due to their useful applications in separation, gas storage, sensing, catalysis, and in biomedicine (Arima et al., 2015; Han et al., 2018). Assembled mostly using metals and non-toxic $\gamma$-CDs widely used for biomedical applications(Loftsson and Brewster, 1996; Loftsson and Duchêne, 2007), they advantageously display high surface areas, adjustable chemical functionality, and structural diversity (Arima et al., 2015; Han et al., 2018; Qiu et al., 2020; Smaldone et al., 2010; Qiu et al., 2018). CD-MOFs were shown to incorporate a large variety of anticancer (e.g., doxorubicin, fluorouracil, methotrexate, quercetin), anti-inflammatory (e.g., ibuprofen, furbiprofen, fenbufen, ketoprofen), antihypertensive (captopril, azilsartan) drugs, acid pump inhibitor (lansoprazole (LPZ)), and various other pharmaceutical ingredients (e.g., salicylic acid, ferulic acid, pseudolaric acid, folic acid, glycyrrhizic acid, etc) (Liu et al., 2017; Lv et al., 2016; Michida et al., 2015; Moussa et al., 2016; Smaldone et al., 2010; Abuçafy et al., 2018; Rajkumar et al., 2019; Xu et al., 2019). As shown in Fig. 1 upper panel, CD-MOFs de-

\footnotetext{
* Corresponding author.

E-mail address: ruxandra.gref@universite-paris-saclay.fr (R. Gref)
} 

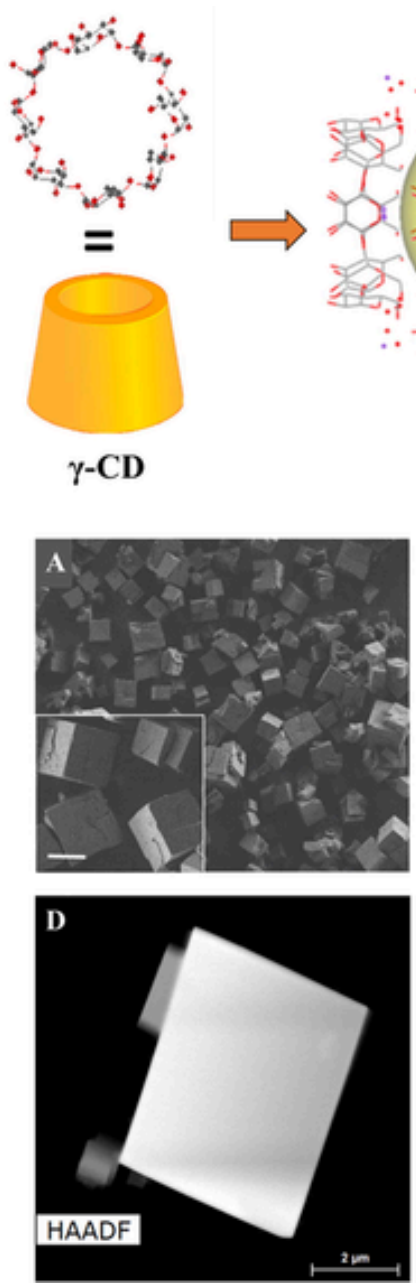

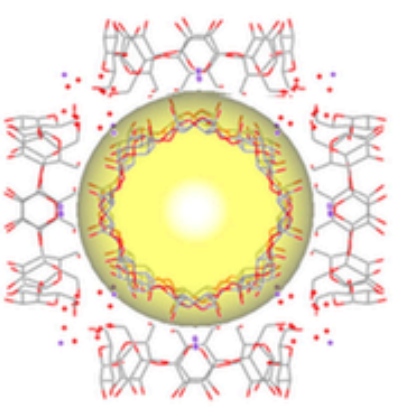

CD-MOF

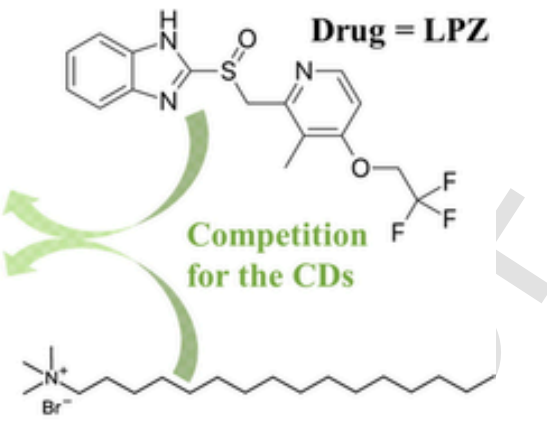

Surfactant $=$ CTAB
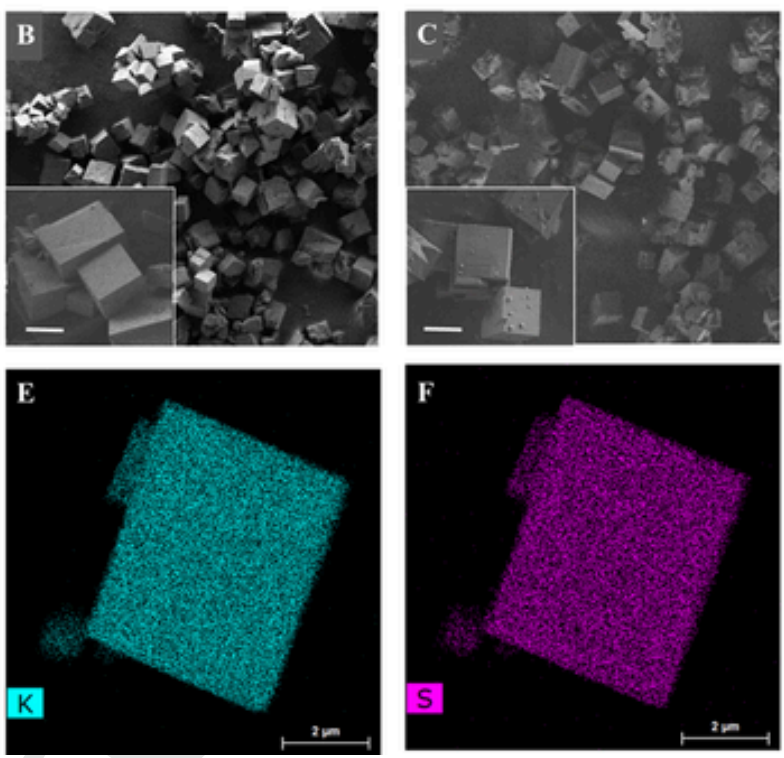

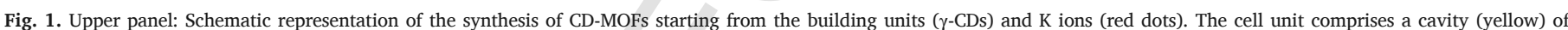

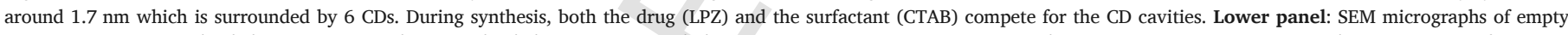

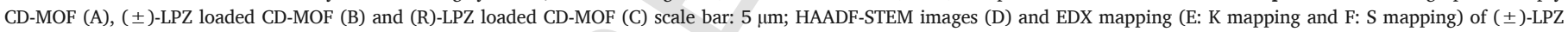
loaded CD-MOF crystals. (For interpretation of the references to colour in this figure legend, the reader is referred to the web version of this article.)

limit an extended framework incorporating the symmetric cyclic $\gamma$-CD oligosaccharide, and are based on the coordination of the $\mathrm{K}^{+}$alkali metal cations to alternating $\alpha$-1,4-linked D-glucopyranosyl units on the primary and secondary faces of the $\gamma$-CD tori (Smaldone et al., 2010). Drugs were mainly loaded by impregnation inside the porosity and loadings reached at best $12.6 \mathrm{wt} \%$ in the case of captopril (Liu et al., 2016). The loadings were increased when the molecules of interest were co-crystallized with CD-MOFs. For instance, rhodamine B could be incorporated with loadings of $18.5 \mathrm{wt} \%$ (Smaldone et al., 2010).

One remarkable result was the incorporation of LPZ in CD-MOFs with loadings as high as $23 \pm 2 \mathrm{wt} \%$ (Li et al., 2017). The crystallized particles of around $6 \mu \mathrm{m}$ were prepared using cetyltrimethyl ammonium bromide (CTAB) as stabilizer and had regular shapes (Furukawa et al., 2012). When CD-MOFs were prepared in the absence of CTAB, they had irregular shapes and high polydispersity, but the LPZ loadings were still in the range $21-23 \mathrm{wt} \%$. The high LPZ loadings correspond to a 1:1 M ratio between LPZ and $\gamma$-CDs in the matrix. However, the role played by CTAB in building regular crystalline supramolecular structures without affecting drug loading is still to be uncovered. Indeed, both CTAB and LPZ compete for the CD cavities inside the CD-MOFs (Fig. 1, upper panel). Moreover, LPZ is a fragile molecule which readily degrades in both acidic (DellaGreca et al., 2006; Ekpe and Jacobsen, 1999; Gupta et al., 2007) and basic conditions (Battu and Pottabathini, 2015; DellaGreca et al., 2006; Liu et al.,
2005), even during storage at $4{ }^{\circ} \mathrm{C}$ (DiGiacinto et al., 2000). It was interesting to study here whether the integrity of the drug was preserved upon incorporation in CD-MOFs and long-term storage.

To gain information on LPZ chemical stability as well as LPZ and CTAB respective locations, we employed a set of complementary techniques. First, LPZ distribution inside the CD-MOFs was characterized by scanning transmission electron microscope (STEM) associated with energy-dispersive X-ray spectroscopy (EDX), which is a powerful tool to detect compositional distribution in single particles. In particular, the detection of sulfur (present only in LPZ) enabled studying drug distribution in these slices of CD-MOFs with high resolution. In addition, vibrational circular dichroism (VCD) enabled studying conformational changes of LPZ inside the CD-MOFs.

The ${ }^{1} \mathrm{H}$ nuclear magnetic resonance (NMR) spectroscopy is often used to study CD-drug inclusions in liquid (Cheng et al., 2018; Di Nunzio et al., 2013; Kundu and Roy, 2017; Rajendiran et al., 2014; Ye et al., 2013) or in solid phase (Ferreira et al., 2015; Skorupska et al., 2014; Upreti et al., 2011; Vogt and Strohmeier, 2012; Wulff et al., 2002). Here, although it proved much more challenging, we choose to employ solid-state NMR spectroscopy to directly study the LPZ-loaded in their final state, as crystalline dried powders.

Using state-of-the-art NMR equipment and methods, including high-field magnets (up to $20 \mathrm{~T}$ ), ultra-fast (up to $60 \mathrm{kHz}$ ) magic-angle (MAS) ${ }^{1} \mathrm{H}$ NMR spectroscopy, complemented by ${ }^{1} \mathrm{H}-{ }^{19} \mathrm{~F}-{ }^{13} \mathrm{C}$ triple-resonance 
NMR and ${ }^{15} \mathrm{~N}$ NMR at natural abundance, new atomic-level insights about the interactions between CTAB/LPZ and CD-MOFs, as well as the localization and configuration of CTAB and LPZ in CD-MOFs have been obtained. A special attention has been attributed to the competition of LPZ and CTAB for the CDs in CD-MOFs. These data were further supported by molecular simulation, which allowed comparing the respective interactions between LPZ, CTAB and the CD-MOFs. In a nutshell, the experimental data corroborated the simulations enabling gaining insights on the mechanism of formation and locations of individual constituents.

Finally, thermogravimetric analysis coupled to Fourier transform infrared spectroscopy (TGA-FTIR) was used to investigate the thermal decomposition of LPZ as free drug or incorporated in CD-MOFs. The thermal stability of the drug molecules was preserved after three years storage.

\section{Experimental}

\subsection{Materials and reagents}

The chemical compounds, including $\gamma$ - $\mathrm{CD}$, CTAB, potassium hydroxide $(\mathrm{KOH})$, methanol, isopropanol and ethanol were purchased from Sigma-Aldrich Co. Ltd (France). (R)-LPZ was provided by Toronto Research Chemicals and ( \pm )-LPZ was provided by Zhuhai Rundu Co. Ltd (China). Water was filtered by a Milli-Q system $(18.4 \mathrm{M} \Omega \mathrm{cm}$, Millipore, Milford, MA, USA). All chemicals were used without further purification.

\subsection{Synthesis of CD-MOF crystals with or without $L P Z$}

CD-MOF crystals were synthesized as previously reported (Li et al., 2017). Briefly, CD-MOFs were prepared by reacting $\gamma$-CD $(0.125 \mathrm{mM})$ with $\mathrm{KOH}(200 \mathrm{mM})$. The solution was filtered through a $0.45 \mu \mathrm{m}$ membrane to remove any possible dust. Then, methanol was allowed to diffuse in this reaction mixture placed in a vial at $50{ }^{\circ} \mathrm{C}$ for $6 \mathrm{~h}$. To obtain monodispersed crystals, $6.0 \mathrm{~mL}$ of the supernatant was transferred into another vial with $48.0 \mathrm{mg}$ of CTAB, then the suspension was incubated for $3 \mathrm{~h}$ at room temperature. The crystals were finally harvested, washed three times with isopropanol and dried at $50{ }^{\circ} \mathrm{C}$ overnight under vacuum. LPZ-loaded CD-MOFs crystals were synthesized in a similar way, except that $( \pm)$-LPZ or $(R)$-LPZ $(80 \mathrm{mM})$ was added in the aqueous solution of $\gamma$-CD $(0.125 \mathrm{mM})$ and $\mathrm{KOH}(200 \mathrm{mM})$. After purification, the CD-MOFs loaded or not with LPZ were stored at $4{ }^{\circ} \mathrm{C}$ in closed vials until further use.

The drug payload is defined as the percentage of LPZ in the drug-loaded CD-MOFs and was calculated as Equation:

Payload $(\%)=\frac{\text { Encapsulated Drug }(\mathrm{mg})}{\text { Drug loaded CD }- \text { MOF }(\mathrm{mg})} \times 100$

\subsection{Characterization of CD-MOFs crystals before or after LPZ encapsulation}

The morphologies of all synthesized CD-MOF crystals were observed by scanning electron microscopy (SEM). SEM images were acquired on a Zeiss SUPRA 55 VP field emission gun scanning electron microscope fitted with an EDAX EDS analytical system. It was set to a low voltage $(1 \mathrm{kV})$ and low currant (a few pA) in order not to damage the samples and to avoid any conductive coating that could bother direct observation of the samples. Secondary electron type detector was used to record the images.

STEM associated with elemental chemical Energy-dispersive X-ray spectroscopy (EDX) cartography was employed to characterize the elemental ( $\mathrm{C}, \mathrm{H}, \mathrm{N}, \mathrm{S}$, and $\mathrm{Br}$ ) distribution in the particles. The MOFs samples were placed on a copper grid covered with a pure carbon mem- brane. The observations in STEM were made on a Titan Themis 200 microscope (FEI/Thermo Fischer Scientific) equipped with a geometric aberration corrector on the probe. This microscope was also equipped with the "Super-X" systems for EDX analysis with a detection angle of $0.9 \mathrm{sr}$. The observations were made at $200 \mathrm{kV}$ with a probe current of about $70 \mathrm{pA}$ and a half-angle of convergence of $24 \mathrm{mrad}$. High-angle annular dark-field (HAADF)-STEM images were acquired with a camera length of $110 \mathrm{~mm}$ (inner/outer collection angles were respectively $69 \mathrm{mrad}$ and $200 \mathrm{mrad}$ ).

The synthesized particles were further cut into thin sections for further STEM observations. Briefly, the dried CD-MOFs powder samples were embedded in epoxy resin (Delta microscopie - France) and polymerized $48 \mathrm{~h}$ at $56^{\circ} \mathrm{C}$. Sections were performed in an ultramicrotome (Leica UC6) using a diamond trim (LFG France). The section thickness was between 100 and $200 \mathrm{~nm}$ and the sections were directly collected on 300 mesh carbon grids.

Elemental analysis was performed to quantify the $\mathrm{C}, \mathrm{H}, \mathrm{N}, \mathrm{S}$, and $\mathrm{Br}$ elemental weight contents in the CD-MOFs particles loaded or not with LPZ. LPZ and CTAB powders were used as controls. $\mathrm{N}$ and $\mathrm{S}$ are present only in LPZ but not in CD-MOFs. Moreover, $\mathrm{N}$ and $\mathrm{Br}$ are in CTAB but not in CD-MOFs. Therefore, the amounts of $\mathrm{Br}$ and $\mathrm{N}$ found in empty CD-MOFs was used to quantify CTAB content. The amount of $S$ was used to determine the amount of LPZ, and Br for CTAB in LPZ loaded CD-MOFs. The amounts of $\mathrm{C}, \mathrm{H}$, and $\mathrm{N}$ were used to confirm the results. Briefly, the determination of $\mathrm{C}, \mathrm{H}, \mathrm{N}$ was performed with a combustion method using an Elemental Analyzer (Perkin-Elmer 2400 CHNS/O Series II System). Sample elements were converted to gases at $1050{ }^{\circ} \mathrm{C}$. The quantification of $\mathrm{Br}$ was performed using a combustion method to decompose organic samples. Then a potentiometric titration was applied using Titroprocessor (835 Titrando) and ion chromatography analysis was performed on Dionex IC system. Sulfur was determined by a combustion method, followed by gravimetric titration and ion chromatography analysis (Dionex IC system).Inductively coupled plasma mass spectrometry (ICP-MS) analysis was used to further confirm the payload. After CD-MOFs digestion using aqua regia (15 min under ultrasonic bath), an ICP-MS equipped with a triple quadrupole (Agilent 8800, Agilent Technologies, Japan) was used for the analysis. Operation conditions were daily optimized using a tuning solution. $\mathrm{S}$ isotopes were detected using "mass shift mode" $\left({ }^{48} \mathrm{SO}^{+},{ }^{49} \mathrm{SO}^{+}\right.$and $\left.{ }^{50} \mathrm{SO}^{+}\right)$after their reaction with oxygen in the cell. Dwell time for each of the targeted isotopes was $1 \mathrm{~s}$. S was quantified using external calibration prepared with certified $1000 \mathrm{mg} \mathrm{L}^{-1} \mathrm{~S}$ standard (Merck, Germany).

The vibrational infrared (IR) absorption and VCD spectra of CD-MOFs were measured using a Fourier-transform infrared spectroscopy (FTIR) spectrometer (Vertex 70, Bruker) equipped with a VCD module (PMA 50, Bruker). The IR radiation was filtered by a low-pass filter cutting at $2000 \mathrm{~cm}^{-1}$, linearly polarized, and then modulated by a $50 \mathrm{kHz} \mathrm{ZnSe}$ photo-elastic modulator (Hinds). The signal was measured by a Mercury-Cadmium-Telluride (MCT) IR detector equipped with a $\mathrm{BaF}_{2}$ window and cooled with liquid nitrogen then demodulated using a lock-in amplifier (Stanford Research Systems SR 830). A spectral resolution of $4 \mathrm{~cm}^{-1}$ was used for both absorption and VCD spectra. The alignment of the spectrometer was controlled by checking the mirror-image relation between the VCD spectra of the two enantiomers of camphor $\left(0.3 \mathrm{M}\right.$ in $\left.\mathrm{CCl}_{4}\right)$. The samples were prepared by mixing the studied system with $\mathrm{KBr}$ with a mortar and pestle and pressing $200 \mathrm{mg}$ pellets. $\mathrm{KBr}$ was first grinded in a mixer mill (MM 400 Retsch) at $20 \mathrm{~Hz}$ during $30 \mathrm{~min}$ and kept dry in an oven at $80^{\circ} \mathrm{C}$, as usually done for the studies of solid samples (Declerck et al., 2019; Pérez-Mellor and Zehnacker, 2017). Two concentrations were used for each system, with the following amounts for $1 \mathrm{~g}$ of $\mathrm{KBr}$ : 3.8 or $4.3 \mathrm{mg}$ for LPZ, 2.5 or $5 \mathrm{mg}$ for bare CD-MOF, and 4.5 or $5.5 \mathrm{mg}$ for CD-MOF loaded with LPZ. The artefacts due to the birefringence were eliminated following 
the procedure proposed by Merten (Merten et al., 2008), derived from that introduced by Buffeteau (Buffeteau et al., 2005). It consists in rotating the sample in the plane perpendicular to the light propagation axis for each side of the pellet and averaging the spectra obtained for the pellets at $0^{\circ}, 90^{\circ}, 180^{\circ}, 270^{\circ}$. The measurements were repeated twice. The final spectrum was obtained by adding 2100 scans for each sample position and averaging all the positions so that the total acquisition time was $4 \mathrm{~h}$.

\subsection{Solid-state NMR characterization of CD-MOFs crystals with or without} $L P Z$

The ${ }^{1} \mathrm{H} \rightarrow{ }^{19} \mathrm{~F} \rightarrow{ }^{13} \mathrm{C}$ cross polarized magic angle spinning (CPMAS) NMR spectra were recorded on a Bruker $500 \mathrm{MHz}$ WB NMR spectrometer using a ${ }^{1} \mathrm{H}^{19}{ }^{19} \mathrm{~F}-\mathrm{X}$ triple-resonance $2.5 \mathrm{~mm}$ probe (Martineau et al., 2011). The initial ${ }^{1} \mathrm{H} \rightarrow{ }^{19} \mathrm{~F}$ CP was used to reduce the repetition delay, taking advantage of short $\mathrm{T}_{1}\left({ }^{1} \mathrm{H}\right)$ compared to $\mathrm{T}_{1}\left({ }^{19} \mathrm{~F}\right.$ ) (Yu et al., 2015). The ${ }^{13} \mathrm{C} C \mathrm{CP}$ experiments were recorded on a $9.4 \mathrm{~T}$ magnet $\left({ }^{1} \mathrm{H}\right.$ and ${ }^{13} \mathrm{C}$ Larmor frequency of 400 and $100 \mathrm{MHz}$, respectively) with a Bruker spectrometer, using a $4 \mathrm{~mm}$ double resonance MAS probe. The ${ }^{13} \mathrm{C}$ chemical shifts are referenced to TMS. The contact time was set to $3.5 \mathrm{~ms}$, the recycle delay was set at $3 \mathrm{~s}$, the initial $90^{\circ}$ pulse on ${ }^{1} \mathrm{H}$ to $2.5 \mu$ s with a Radio Frequency (RF) field of $70 \mathrm{kHz} .{ }^{1} \mathrm{H}$ SPINAL-64 decoupling was applied during the ${ }^{13} \mathrm{C}$ acquisition. The ${ }^{1} \mathrm{H}$ MAS NMR spectra were recorded at a magnetic field of $18.8 \mathrm{~T}$, using a Bruker $850 \mathrm{MHz}$ WB NMR spectrometer and a HXY $1.3 \mathrm{~mm}$ probe in double-resonance mode. The spectra were acquired using Hahn echo pulse sequence, with a $90^{\circ}$ pulse duration of $2.5 \mu \mathrm{s}$, an inter-pulse delay synchronized with one rotor period and a spinning rate of $60 \mathrm{kHz}$. The recycle delay was set to $5 \mathrm{~s}$ and 16 transients were recorded for each sample. The ${ }^{1} \mathrm{H}$ chemical shifts were referenced to TMS. The ${ }^{1} \mathrm{H}_{-}{ }^{1} \mathrm{H} 2 \mathrm{D}$ MAS exchange NMR experiment was recorded with a recycle delay of $2 \mathrm{~s}$ and two different mixing times ( 5 and $10 \mathrm{~ms}$ ). $700 \mathrm{t}_{1}$ slices with 16 transients were co-added. The States procedure provides a phase sensitive 2D NMR spectrum.

${ }^{15} \mathrm{~N}$ CPMAS NMR spectra were recorded on a Bruker 400 WB NMR spectrometer using a $4 \mathrm{~mm}$ probe head and spinning at $10 \mathrm{kHz} .10,240$ and 75,776 transients were accumulated for LPZ and LPZ loaded CD-MOF, respectively. Similar ${ }^{15} \mathrm{~N}$ CPMAS NMR spectra were recorded at $8 \mathrm{kHz}$ MAS frequency on a Bruker 600 SB NMR spectrometer using a novel cryogenically cooled $3.2 \mathrm{~mm}$ HCN CPMAS probe (Biosolids CryoProbe $^{\mathrm{TM}}$ ) (Hassan et al., 2020). 528 and 122888 transients were accumulated for LPZ and LPZ loaded CD-MOF, respectively. In all cases, acquisition time is $20 \mathrm{~ms}$. No apodization was applied on the LPZ spectrum, while a $30 \mathrm{~Hz}$ Lorentzian apodization was applied on ${ }^{15} \mathrm{~N}$ CPMAS NMR spectrum of LPZ loaded CD-MOFs. All the samples were finely ground into powders and packed into a zirconia rotor of the appropriate size. The NMR spectra were acquired using TopSpin 3.5 Bruker Software and processed with the DMfit program (Massiot et al., 2002).

\subsection{Molecular simulation}

The crystal structure of CD-MOFs was extracted from the single crystal structure of MOF-1 as reported in the literature (Forgan et al., 2012). In the docking model, an expanded non-periodic structure was used, where the $\mathrm{OH}^{-}$ion was replaced by $\mathrm{H}_{2} \mathrm{O}$ and the $\mathrm{K}^{+}$ion was deleted in PyMOL 2.2.0. The molecular structure of $\gamma$-CD was extracted from reported CD-MOFs structures (Forgan et al., 2012). The structure of CTAB was obtained from the PubChem of National Center for Biotechnology Information (PubChem CID: 5974; https://pubchem.ncbi.nlm. nih.gov). AutoDock was then applied using docking program AutoDock Vina 1.1.2 (Allouche, 2012) to optimize their molecular structures.
Lamarckian Genetic Algorithm (LGA) in combination with a grid-based energy evaluation method was used to pre-calculate grid maps according to the interatomic potentials of all atom types present in the host and guest molecules, including the Lennard-Jones potentials for van der Waals interactions and Coulomb potentials for electrostatic interactions. A grid map of dimensions $30 \AA \times 30 \AA \times 30 \AA$ and $10 \AA \times 10 \AA \times 10 \AA$, with a grid spacing of $0.375 \AA$, was placed to cover the CD-MOF and $\gamma$-CD models, respectively. With the help of AutoDockTools (Zhao and Sanner, 2007), the atomic partial charges were calculated by the Gasteiger-Marsili method (Gasteiger and Marsili, 1980). The parameters used for the global search were an initial population of 50 individuals, with a maximal number of energy evaluations of 1,500,000 and a maximal number of generations of 50,000 as an end criterion. Other docking parameters were set as default.

\subsection{Thermal degradation analysis}

Thermal decomposition of LPZ loaded CD-MOFs was investigated using a PerkinElmer thermogravimetric analysis Fourier transform infrared spectroscopy (TGA-FTIR) system which consists of a TGA 4000 coupled to a FTIR (Spectrum Two) by TL8000 interface. Samples masses ranging from 6 to $10 \mathrm{mg}$ were heated from 25 to $600{ }^{\circ} \mathrm{C}$ at a rate of $10{ }^{\circ} \mathrm{C} / \mathrm{min}$ in a $\mathrm{N}_{2}$ atmosphere. The flow rate was approximately $60 \mathrm{~mL} /$ min applied by the TGA 4000 internal mass flow controller and further monitored by TL8000 to ensure the constant flow rate and avoid the gas mixing in the cell. The heated line that transfers evolved gases from the TGA to the FTIR was set at $260{ }^{\circ} \mathrm{C}$ and the IR gas cell was held at $270{ }^{\circ} \mathrm{C}$. IR spectra were recorded with a $4 \mathrm{~cm}^{-1}$ resolution in the range of $4,000-500 \mathrm{~cm}^{-1}$. Pure LPZ and CD-MOF particles were used as controls.

\section{Results and discussions}

\subsection{Synthesis and characterization of LPZ loaded CD-MOFs}

CD-MOFs crystals were successfully obtained displaying a crystalline structure with regular cubic morphology and diameters around $5 \mu \mathrm{m}$ (Fig. 1A), in agreement with previously reported data (Li et al., 2017). The $( \pm)$-LPZ or $(R)$-LPZ loaded CD-MOFs were prepared with a similar procedure except the addition of the drug directly in the reaction mixture composed of $\gamma-\mathrm{CD}, \mathrm{KOH}$ and CTAB.

LPZ was reported to degrade rapidly within $6-8 \mathrm{~h}$ in basic conditions, especially at high temperature (e.g. $60{ }^{\circ} \mathrm{C}$ (Battu and Pottabathini, 2015) or $80{ }^{\circ} \mathrm{C}$ (Shankar et al., 2017)). In this study, LPZ was incorporated in $\mathrm{CD}-\mathrm{MOF}$ at lower temperature $\left(50{ }^{\circ} \mathrm{C}\right)$ and during a $6 \mathrm{~h}$ procedure, in the presence of $\mathrm{KOH}(200 \mathrm{mM})$. LPZ was extracted from the prepared CD-MOFs and its integrity was assessed by HPLC, showing exactly the same retention time peak without the appearance of degradation products, out ruling a possible degradation during CD-MOF preparation. Possibly, CDs played a protective role, as it has been observed in previous studies (Pasic, 2008).

Three complementary methods were used to assess drug loading: i) elemental analysis (determination of "N" and " $\mathrm{S}$ " contents in loaded samples) showed that the ( \pm )-LPZ payload reached $21 \pm 2 \mathrm{wt} \%$ (Table 1); ii) HPLC-UV-vis allowed determining payloads of $22 \pm 2 \mathrm{wt} \%$ and iii) ICP-MS showed payloads of $20 \pm 2 \mathrm{wt} \%$. It was concluded that there is a good agreement between the three methods. Moreover, no significant drug loading differences were observed when $(R)$-LPZ was incorporated instead of $( \pm$ )-LPZ (less than $2 \%$ differences, as quantified by ICP-MS, showing (R)-LPZ payload of $20 \pm 1.5 \mathrm{wt} \%$ ). Interestingly, despite the high LPZ loadings, the CD-MOFs' morphology and size were not affected by LPZ incorporation (Fig. 1B and C).

HAADF-STEM images confirmed the regular morphologies of the CD-MOFs possessing sharp edges, loaded or not with the drug (Fig. 1D). When coupled with EDX, this method gave insights on the element 
Table 1

Elemental analysis of CD-MOFs loaded or not with LPZ. Free LPZ and CTAB were used as controls.

\begin{tabular}{|c|c|c|c|c|c|c|c|c|c|}
\hline Sample & C (\%) & H (\%) & S (\%) & & N (\%) & $\mathrm{Br}(\%)$ & LPZ (\%) & & СТАВ (\%) \\
\hline & $A^{a}$ & A & A & $\mathrm{B}^{\mathrm{b}}$ & A & B & A & B & \\
\hline LPZ & $51.8 \pm 0.4$ & $3.3 \pm 0.3$ & $9.1 \pm 0.3$ & $8.7 \pm 0.3$ & $11.15 \pm 0.3$ & - & $98 \pm 3(\mathrm{~N})$ & $100 \pm 3(S)$ & - \\
\hline СТАВ & $62.9 \pm 0.4$ & $12.2 \pm 0.3$ & - & - & $3.8 \pm 0.1$ & $22.4 \pm 0.5$ & - & - & $\begin{array}{l}100 \pm 3(\mathrm{~N}) \\
101 \pm 2(\mathrm{Br})\end{array}$ \\
\hline CD-MOF & $42.4 \pm 0.4$ & $6.8 \pm 0.3$ & - & - & $0.6 \pm 0.1$ & $3.1 \pm 0.3$ & - & - & $\begin{array}{l}14.8 \pm 3(\mathrm{~N}) \\
14.1 \pm 2(\mathrm{~S})\end{array}$ \\
\hline LPZ loaded CD-MOF & $42.0 \pm 0.4$ & $5.5 \pm 0.3$ & $2.0 \pm 0.3$ & $2.1 \pm 0.1$ & $2.6 \pm 0.1$ & $0.3 \pm 0.1$ & $23 \pm 3(S)$ & $24 \pm 3(S)$ & $\begin{array}{l}0.05 \pm 0.02(\mathrm{~N}) \\
1.4 \pm 0.5(\mathrm{Br})\end{array}$ \\
\hline
\end{tabular}

A: The experiments were performed with a combustion method using an elemental analyzer; B: The results were obtained by ICP-MS.

distribution inside the particles. The homogeneous distribution of $\mathrm{K}$ in the CD-MOFs (Fig. 1E) indicates their homogeneity in terms of composition. Indeed, sulfur (S) is only present in LPZ and not in the CD-MOFs matrix. Fig. 1F shows the high homogeneity in terms of drug distribution in the particles. Control samples (Fig. S1) shows that there is no residual $\mathrm{S}$ in the empty $\mathrm{CD}-\mathrm{MOF}$. Fluor $(\mathrm{F})$ is a lighter element compared to S, present in LPZ and not in CD-MOFs. Fig. S2 shows that this element diffracted more at the particle edges in thick samples, as confirmed also by the mapping of $\mathrm{C}$ and $\mathrm{O}$.

Thus, to circumvent possible artefacts due to the thickness of the samples and gain deeper insights on the element distribution inside the CD-MOFs, these were cut into slices of 100-200 nm thick and analyzed again by HAADF-STEM. Fig. S3 shows the mapping of all the CD-MOF components (F, C, O, S, K), supporting the homogeneous drug distribution within their structure.

It has been previously reported (Li et al., 2017) by some of us that the Raman spectra of different individual CD-MOFs particles perfectly superimposed, suggesting the homogenous composition of the drug loaded CD-MOFs particles. However, element mapping (Fig. S3) gave even deeper and clearer insight.

As reported by Furukawa et al (Furukawa et al., 2012), the addition of CTAB during the synthesis of the particles is needed to control their size and achieve regular cubic morphologies. However, no study has been performed yet to elucidate the possible interference of CTAB with drug loading inside CD-MOFs. To do so, CTAB residual amounts in empty and loaded CD-MOFs was determined by both elemental analysis and ICP-MS (Table 1).

As described in materials and method section, СТAB contains $\mathrm{Br}$ and $\mathrm{N}$, whereas $\mathrm{CD}-\mathrm{MOF}$ do not. Elemental analysis of control samples (CTAB and LPZ) shows that their composition can be retrieved from elemental analysis data (Table 1). Therefore, the accurate quantification of $\mathrm{Br}$ and $\mathrm{N}$ in the synthesized CD-MOFs allows for determining their residual CTAB content.

The amount of CTAB in empty CD-MOF crystals was as high as $15 \pm 1.4 \mathrm{wt} \%$ (corresponding to molar ratio of $2: 3$ between $\mathrm{CTAB}$ and $\gamma$-CD). This suggests that CTAB was incorporated in high amounts in $\mathrm{CD}-\mathrm{MOF}$, possibly poisoning crystals growing and thus allowing for the formation of small crystals. Surprisingly, the amount of residual CTAB in LPZ-loaded CD-MOFs was practically undetectable (less than $0.1 \mathrm{wt} \%$ ) despite the fact that the same amount of CTAB was used in the preparation procedure as in the case of empty CD-MOFs.

In contrast, the contents of LPZ in the CD-MOFs were similar, whether $\mathrm{CTAB}$ was used or not in their preparation procedure (payloads of $21 \pm 2 \mathrm{wt} \%$ and $23 \pm 2 \mathrm{wt} \%$, respectively).

It can be concluded that the addition of LPZ during the CD-MOF formation competed with CTAB, and LPZ substituted CTAB in the final particle.

\subsection{Solid-state NMR characterization}

Magic-angle spinning (MAS) solid-state NMR spectroscopy measurements were performed in order to get further information about the interaction of CTAB and CD-MOF as well as the modification of LPZ structure upon encapsulation.

\subsubsection{Solid-state NMR characterization of CD-MOF}

Fig. 2 displays the $1 \mathrm{D}{ }^{1} \mathrm{H}$ and ${ }^{13} \mathrm{C}$ solid-state NMR spectra of pristine $\gamma$-CD and of the CD-MOF. The ${ }^{1} \mathrm{H}$ MAS spectrum of $\gamma$-CD shows a single broad featureless resonance while the spectrum of the CD-MOF, acquired under the same experimental conditions, contains three sets of peaks: the hydroxyl protons at $5.20 \mathrm{ppm}$, the $\mathrm{CH}$ groups and the $\mathrm{CH}_{2}$ groups at 3.49 and $3.06 \mathrm{ppm}$, respectively (Bekiroglu et al., 2003). ${ }^{1} \mathrm{H}_{-}{ }^{13} \mathrm{C}$ 2D NMR experiments validate our line assignment (Fig. S5).

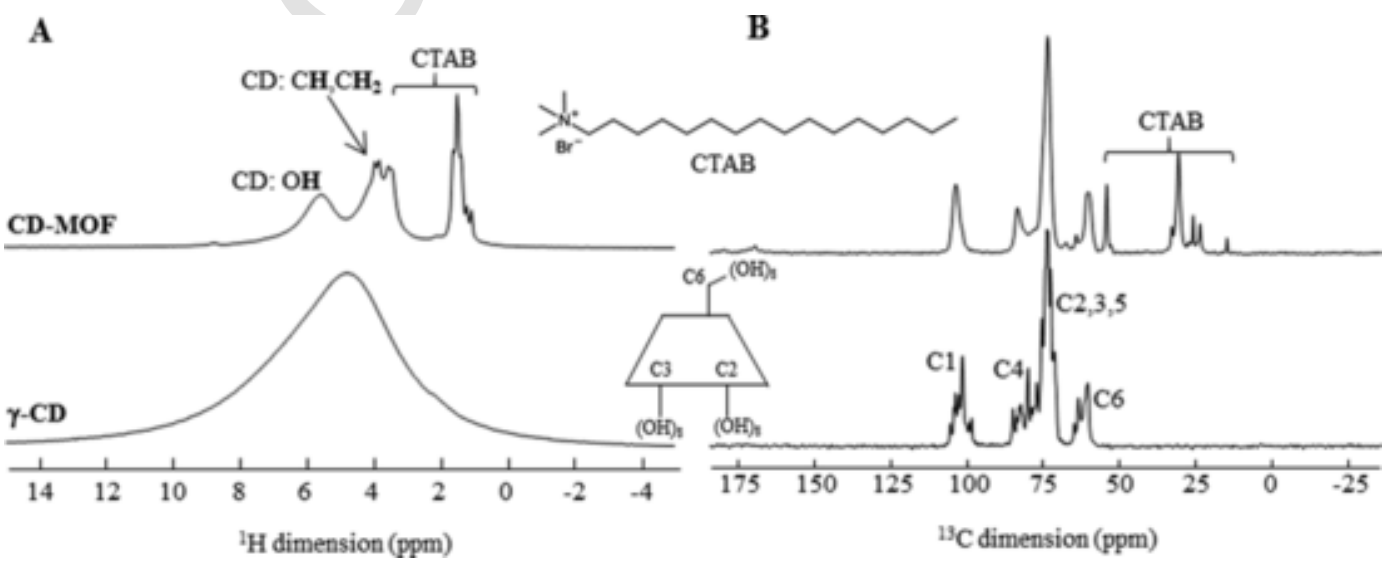

Fig. 2. ${ }^{1} \mathrm{H}$ MAS (A) and ${ }^{13} \mathrm{C}$ CPMAS (B) NMR spectra of CD-MOF (top) and $\gamma$-CD (bottom). The CTAB and CD molecules are shown on the right. The NMR lines are assigned. 
The lines corresponding to the $\gamma$-CD appear narrower on the CD-MOF. This might be due to the intrinsic porosity of the CD-MOF, which increases the ${ }^{1} \mathrm{H}-{ }^{1} \mathrm{H}$ distances, hence reduces the homonuclear dipolar interaction and the linewidth. An additional set of peaks is noticed at $1 \mathrm{ppm}$ which corresponds to the aliphatic protons of the CTAB used in the CD-MOF synthesis and trapped in their final structure. This is confirmed by the ${ }^{13} \mathrm{C}$ CPMAS NMR spectrum, which contains, in addition to the peaks of the $\gamma$-CD (at 102.2, 82.3, 73.5 and $61.8 \mathrm{ppm}$ ), peaks of smaller intensity that correspond to the CTAB. This validates the incorporation of CTAB in the CD-MOFs detected by the elemental analysis (Table 1).

${ }^{1} \mathrm{H}-{ }^{1} \mathrm{H}$ 2D MAS NMR homonuclear correlation spectra were recorded to get further insights into the possible location of the CTAB in the CD-MOFs. The 2D ${ }^{1} \mathrm{H}-{ }^{1} \mathrm{H}$ NMR spectrum of the CD-MOFs recorded with a short mixing time (Fig. $3 \mathrm{~A}$ ) contains cross-correlation peaks of high intensity between the CTAB and the $\mathrm{CH} / \mathrm{CH}_{2}$ of the $\gamma$-CD, while the intensity of the cross-peaks between the $\mathrm{CTAB}$ and the $\mathrm{OH}$ of the $\gamma$-CD are of a much smaller intensity. This is due to shorter distance of the CTAB with the inside protons of the $\gamma$-CD, which indicates that part of the CTAB is very likely located inside the $\gamma$-CD cavity.

\subsubsection{Solid state NMR characterization of LPZ loaded CD-MOF}

The ${ }^{1} \mathrm{H}$ and ${ }^{13} \mathrm{C}$ MAS NMR spectra of LPZ loaded CD-MOFs were displayed in Fig. 4 along with those of pure LPZ. The first observation was that the intensity of the CTAB resonances has strongly decreased (in the $25 \mathrm{ppm}$ region and slightly above $50 \mathrm{ppm}$ ), indicating that the drug loading process has expelled most CTAB molecules from the system, as observed by elemental analysis (Table 1 ).

Several ${ }^{13} \mathrm{C}$ NMR experiments were performed to identify and quantify the various carbon atoms (Fig. S6): a CP with short contact time $(50 \mu \mathrm{s})$ allows selection of the proton bearing carbon atoms while a quantitative CP (Johnson and Schmidt-Rohr, 2014) allows the determination of the number of carbon atoms contributing to each ${ }^{13} \mathrm{C}$ resonance (which can arise from the overlap of several $\mathrm{C}$ atoms). Careful analysis of these experiments showed that all ${ }^{13} \mathrm{C}$ resonances of the drug are found in the LPZ loaded CD-MOF sample, supporting he HPLC data which showed that the drug remained intact after the encapsula-
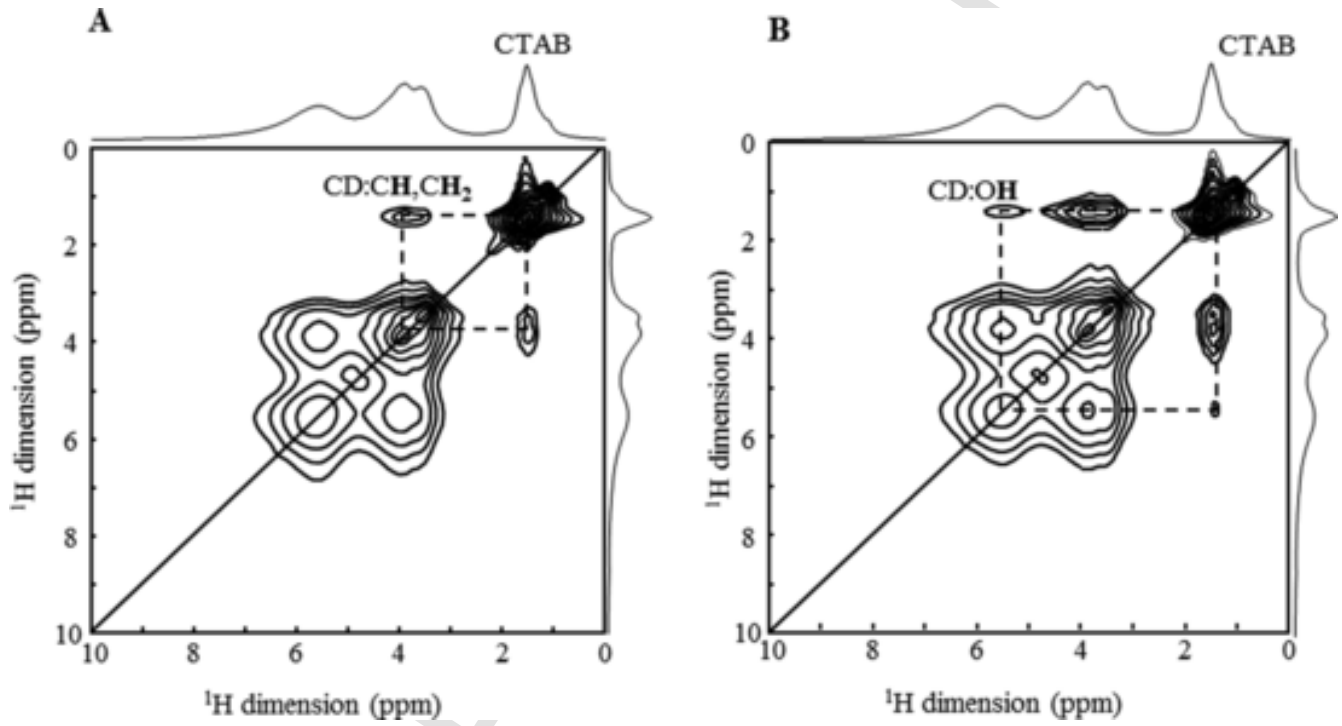

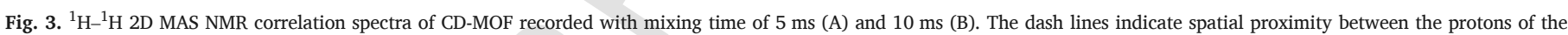
$\mathrm{CTAB}$ and those of the $\gamma$-CD.

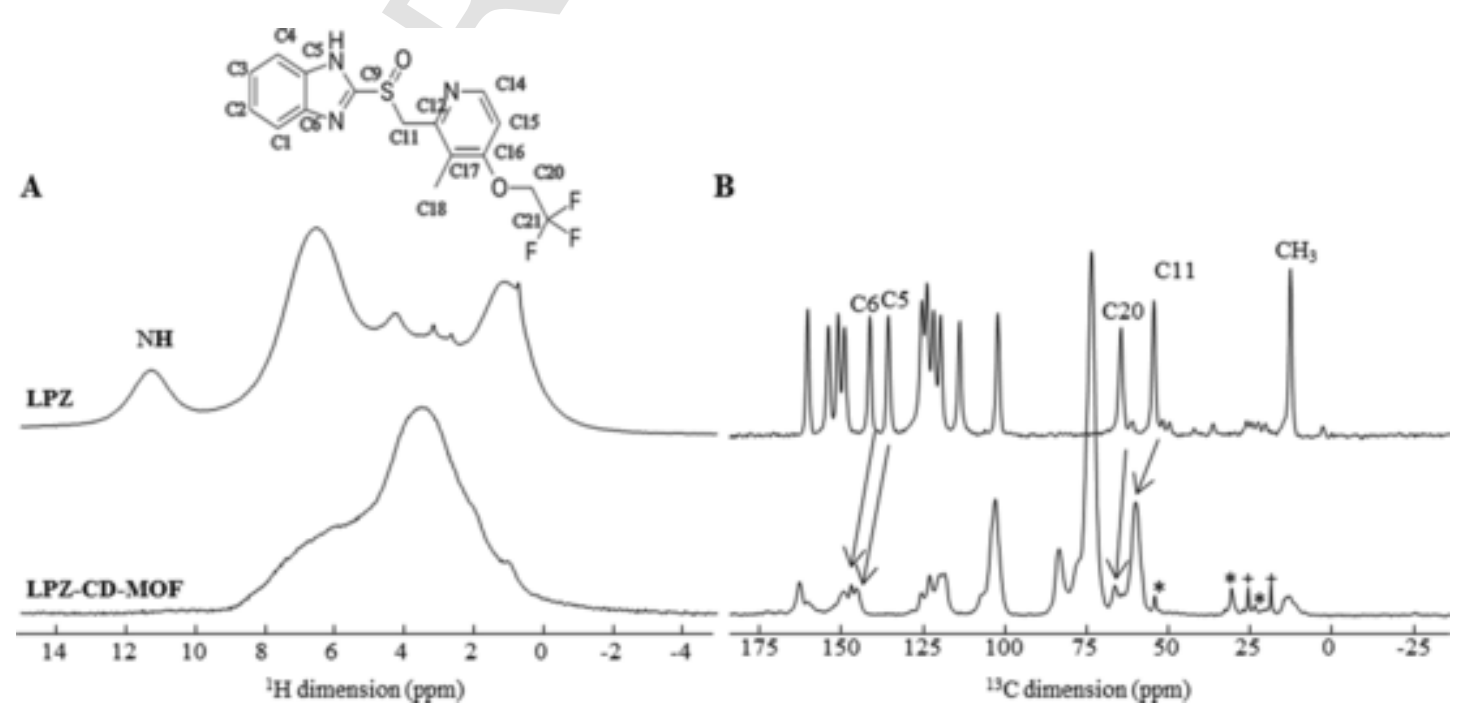

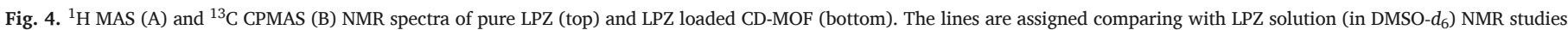
(Table S1) (Ramulu et al., 2013). The molecule of LPZ with carbon atoms numbered is shown. * indicate traces of CTAB together with traces of ethanol. 
tion process, presumably because it was stabilized inside the MOF particles. Stability of LPZ inside the particles is further supported by the ${ }^{13} \mathrm{C}$ liquid-state NMR spectrum of the CD-MOF dissolved in $\mathrm{D}_{2} \mathrm{O}$, which shows the absence of degraded LPZ (Fig. S4).

Finally, the quantitative analysis ${ }^{1} \mathrm{H}-{ }^{1} \mathrm{H}$ and ${ }^{1} \mathrm{H}-{ }^{13} \mathrm{C}$ 2D NMR data (Fig. S7) indicate a ratio between the LPZ and $\gamma$-CD close to 1:1, corroborating the ICP-MS, elemental analysis and HPLC data.

One can notice that the ${ }^{13} \mathrm{C}$ resonances corresponding to the carbon atoms C11 and C20 (overlapped with one CD resonance) of the LPZ have different chemical shifts in the pure and loaded drug (Figs. 4 and 5). Notably, $\mathrm{C} 11$ and $\mathrm{C} 20$ are flexible $\mathrm{sp}^{3}$ carbon atoms, the observed

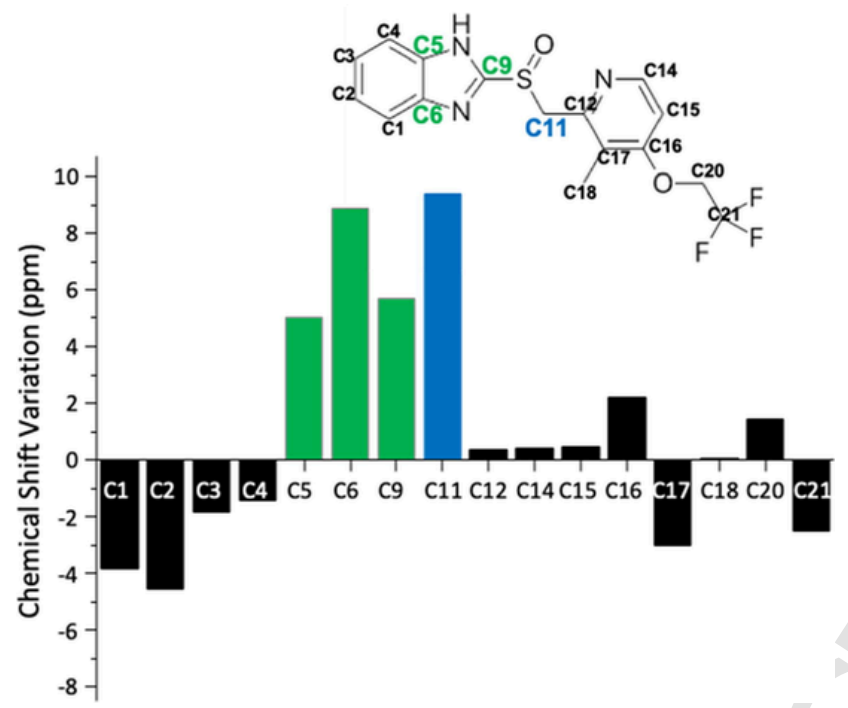

Fig. 5. ${ }^{13} \mathrm{C}$ chemical shift variation (ppm) of the carbon atoms of LPZ pure and loaded in the CD-MOFs. shifts hence very likely correspond to a bending of the drug once it is loaded in the CD-MOFs.

Besides $\mathrm{C} 11$ and C20, C5 and C6, which belong to the imidazole ring, also show significant shift of their ${ }^{13} \mathrm{C}$ resonance upon loading. These shifts are correlated to the disappearance of the $\mathrm{N}-\mathrm{H}$ proton of the pure drug $\left({ }^{1} \mathrm{H}\right.$ chemical shift of 11.5 ppm Fig. 4A), when it is loaded in the $\mathrm{CD}-\mathrm{MOF}$ and with a strong shift of the ${ }^{15} \mathrm{~N}$ resonance in the ${ }^{15} \mathrm{~N}$ CPMAS NMR spectrum (Fig. 6). All these features indicated that the LPZ molecules are deprotonated when loaded in the CD-MOF, possibly because of their basic inner microenvironment.

Despite the relatively small amount of drug loaded (below 25\%) and broadness of the signals (due to chemical shift distributions), the ${ }^{15} \mathrm{~N}$ CPMAS NMR spectrum of the drug-loaded samples could be obtained at natural abundance in less than $48 \mathrm{~h}$ due to significant signal enhancement provided by the novel cryogenically cooled $3.2 \mathrm{~mm} \mathrm{HCN}$ CPMAS probe (see experimental section), while no signal could be obtained in a week in an usual NMR spectrometer (Fig. S8). In formulated drugs, significant ${ }^{15} \mathrm{~N}$ NMR sensitivity gain have been obtained using dynamic nuclear polarization (DNP-MAS) NMR (Ni et al., 2017). However, CD-MOFs are unstable in the solvents that are required for performing DNP-MAS studies. Hence, the cryoprobe technology is so far the only technology that allows probing the state of a low API content in formulations that are not amenable to DNP-MAS NMR. Note that the CPMAS CryoProbe has been used so far for biomolecules (Hassan et al., 2020), and we reported here its first use in the field of materials science.

Despite using a high magnetic field $(20.0 \mathrm{~T})$ and fast MAS $(60 \mathrm{kHz})$, the ${ }^{1} \mathrm{H}$ MAS NMR spectrum of LPZ loaded CD-MOFs only showed broad peaks with extremely low resolution (Fig. 4A and S9), hence $2 \mathrm{D}^{1} \mathrm{H}-{ }^{1} \mathrm{H}$ NMR appeared of limited use. Therefore, to get insights into the interactions between the drug and the CD-MOFs, we used the ${ }^{19} \mathrm{~F}$ nucleus as a spy. The advantage of ${ }^{19} \mathrm{~F}$ is that there is only one fluorine atom in the compound, which belongs to the drug. We therefore recorded, using a dedicated ${ }^{1} \mathrm{H}_{-}{ }^{19} \mathrm{~F}-\mathrm{X}$ probe, a ${ }^{1} \mathrm{H}_{-}{ }^{19} \mathrm{~F}-{ }^{13} \mathrm{C}$ double CPMAS NMR experiment. The initial ${ }^{1} \mathrm{H}_{-}{ }^{19} \mathrm{~F}$ was used to benefit from the shorter relax-

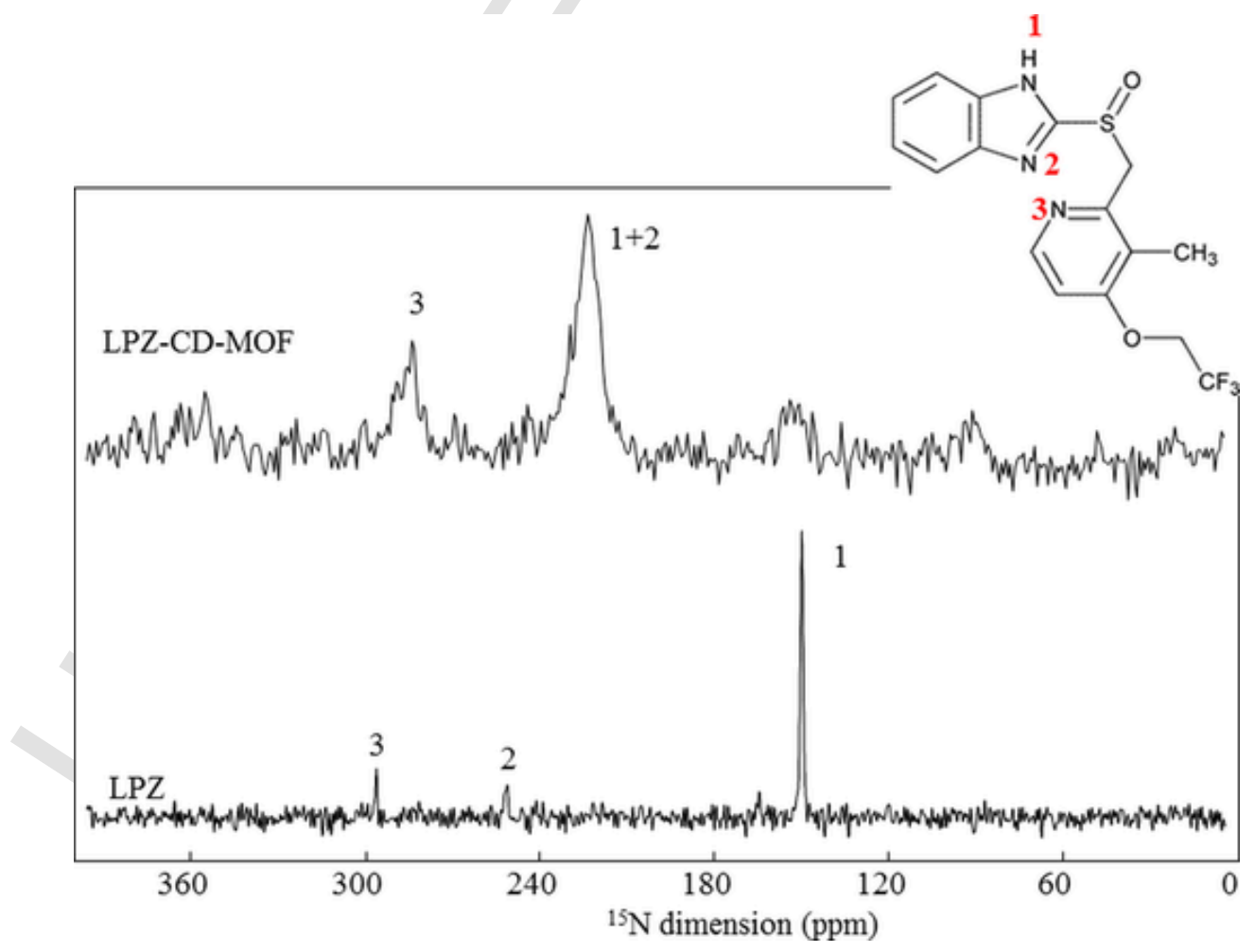

Fig. $6 .{ }^{15} \mathrm{~N}$-CPMAS NMR spectra of pure LPZ and loaded LPZ showing the deprotonation of the imidazole ring upon drug loading in the CD-MOF. 
ation $\mathrm{T}_{1}$ of ${ }^{1} \mathrm{H}$ (shorter than $1 \mathrm{~s}$ ) compared to the one of ${ }^{19} \mathrm{~F}(>5 \mathrm{~s}$ ). This experiment allows the selection of the carbon atoms in the vicinity of the fluorine atom. The resulting ${ }^{13} \mathrm{C}$ NMR spectrum (Fig. 7) was compared to the ${ }^{1} \mathrm{H}-{ }^{13} \mathrm{C}$ CPMAS NMR spectrum, in which all ${ }^{13} \mathrm{C}$ atoms are present (since both the drug and the $\gamma$-CD have protons to transfer magnetization to the carbon atoms). The spectra were normalized to the $\mathrm{CD}$ peak at $73 \mathrm{ppm}$. In the ${ }^{19} \mathrm{~F}_{-}{ }^{13} \mathrm{C}$ CPMAS, one can notice that the $\mathrm{CF}_{3}$ and $\mathrm{C} 20$ carbons of the LPZ have higher intensity, which is an expected result as these are the closest $\mathrm{C}$ atoms to the ${ }^{19} \mathrm{~F}$ nuclei. There is also a significant signal for the ${ }^{13} \mathrm{C}$ of the $\gamma$-CD, indicating its close spatial proximity to the drug. Among the ${ }^{13} \mathrm{C}$ signals of the $\gamma$ - $\mathrm{CD}$, the one labeled $\mathrm{C} 6$ in Fig. 2 (which corresponds to the $\mathrm{CH}_{2} \mathrm{OH}$ ) has higher intensity than the other $\gamma$-CD carbon atoms (Fig. 7). This indicates that the $\mathrm{CF}_{3}$ is in close contact with the $\mathrm{CH}_{2} \mathrm{OH}$ of the $\gamma$ - $\mathrm{CD}$, i.e., the molecule is most probably bend so that the $\mathrm{CF}_{3}$ groups get located outside of the CD cavity.

To investigate the fate of the LPZ molecules once incorporated in CD-MOF crystals, the synthesized particles were characterized by VCD spectroscopy. (R)-LPZ loaded particles were successfully synthesized, reaching the payload of $20 \pm 1.5 \mathrm{wt} \%$. Prior to the VCD spectra, IR spectra was recorded for $(R)$-LPZ, empty CD-MOFs, and drug loaded CD-MOFs. All the bands of CD-MOFs appear unmodified in the IR absorption spectrum of loaded MOF, indicating that the structure of the crystals was preserved after LPZ loading. Several intense bands of (R)-LPZ also appeared in this spectrum, at 1167, 1267 and $1585 \mathrm{~cm}^{-1}$ (Fig. S11). They were not modified in frequency or intensity relative to $(R)$-LPZ. This confirmed the successful drug loading in the particles. In contrast, the region of other intense bands of $(R)-\mathrm{LPZ}$, between the bands at 1040 and $1115 \mathrm{~cm}^{-1}$, was modified in the loaded CD-MOF relative to the pure drug $(R)$-LPZ. This observation points to a modification of the structure of LPZ upon inclusion in the CD-MOFs.

The VCD spectra of $(R)-\mathrm{LPZ}$, bare CD-MOF, and loaded CD-MOF were recorded. As shown in Fig. 8, the spectrum of $(R)$-LPZ is intense, of the order of $5 \times 10^{-4}$. Indeed, intense VCD spectra are often observed for crystalline samples. Most bands present in the VCD spectrum of the drug-loaded MOF are due to the CD-MOF itself, further proving that the structure of $\mathrm{CD}-\mathrm{MOF}$ was kept intact. For example, the IR absorption band of $(R) \mathrm{LPZ}$ at $1167 \mathrm{~cm}^{-1}$ (shown in Fig. S11) corre- sponds to an intense negative band in the VCD spectrum of (R)-LPZ. Although this band is observed in the IR absorption of loaded CD-MOF, it is absent in its VCD spectrum. These data indicate again that the LPZ structure was changed upon inclusion in CD-MOFs. HPLC and NMR data clearly ruled out a possible degradation of the drug. A possible explanation of VCD data could be LPZ deprotonation, as is suggested by the solid-state NMR data.

\subsection{Molecular simulation}

Molecular simulation was carried out to gain insights into the competition between CTAB and LPZ to insert into the available cavities of CD-MOFs. First, the interaction of the two molecules with free $\gamma$-CDs were studied. Fig. 9 (A,B) shows that both LPZ and CTAB crouch in the hydrophobic $\gamma$-CD cavity [14].

In contrast, inside the CD-MOF structures, both LPZ and CTAB preferentially locate in the $\gamma$-CD pairs of CD-MOF rather than in the cage of CD-MOF (yellow sphere in Fig. $9 \mathrm{C}, \mathrm{D}$ ).

As shown in Fig. 9, part of the CTAB molecule is nonetheless located inside the CD-cavity of CD-MOF, which is in agreement with the ${ }^{1} \mathrm{H}-{ }^{1} \mathrm{H}$ MAS NMR spectra reported in Fig. 4. When one LPZ molecule is inserted in the system, the $\mathrm{CF}_{3}$ group tend to locate outside the $\gamma$-CDs of $\mathrm{CD}-\mathrm{MOF}$, in between two of them, which is also in agreement with the ${ }^{19} \mathrm{~F}-{ }^{13} \mathrm{C}$ CPMAS NMR experiments.

LPZ possessed a stronger interaction free energy with both $\gamma$-CD $\left(-5.3 \mathrm{kcal} \cdot \mathrm{mol}^{-1}\right)$ and $\mathrm{CD}-\mathrm{MOF} \quad\left(-7.5 \mathrm{kcal} \cdot \mathrm{mol}^{-1}\right)$ than CTAB $\left(-2.9 \mathrm{kcal} \cdot \mathrm{mol}^{-1}\right.$ and $-4.0 \mathrm{kcal} \cdot \mathrm{mol}^{-1}$ for $\gamma$-CD and CD-MOF, respectively).

In summary, the interaction free Gibbs energy of CTAB is weaker than that of LPZ either with CD-MOF or with $\gamma$-CD, which explains the experimental observations that LPZ molecules are preferentially incorporated in the CD-MOFs when both CTAB and LPZ are used for their preparation. This is likely due to the presence of more nonpolar alkyl groups in CTAB than in LPZ. Moreover, simulations were performed to study the competition between LPZ and CTAB molecules for the CD cavities in CD-MOFs. To do so, one LPZ molecule and one CTAB molecule was placed in contact with one CD Inside the CD-MOF. Based on the free Gibbs energies $\Delta G$ and the Eq. (1), at a temperature of $300 \mathrm{~K}$, with $R=8.314 \mathrm{~J} \mathrm{~mol}^{-1} \mathrm{~K}^{-1}$ and $e=2.71828$, the probability that $\mathrm{LPZ}$

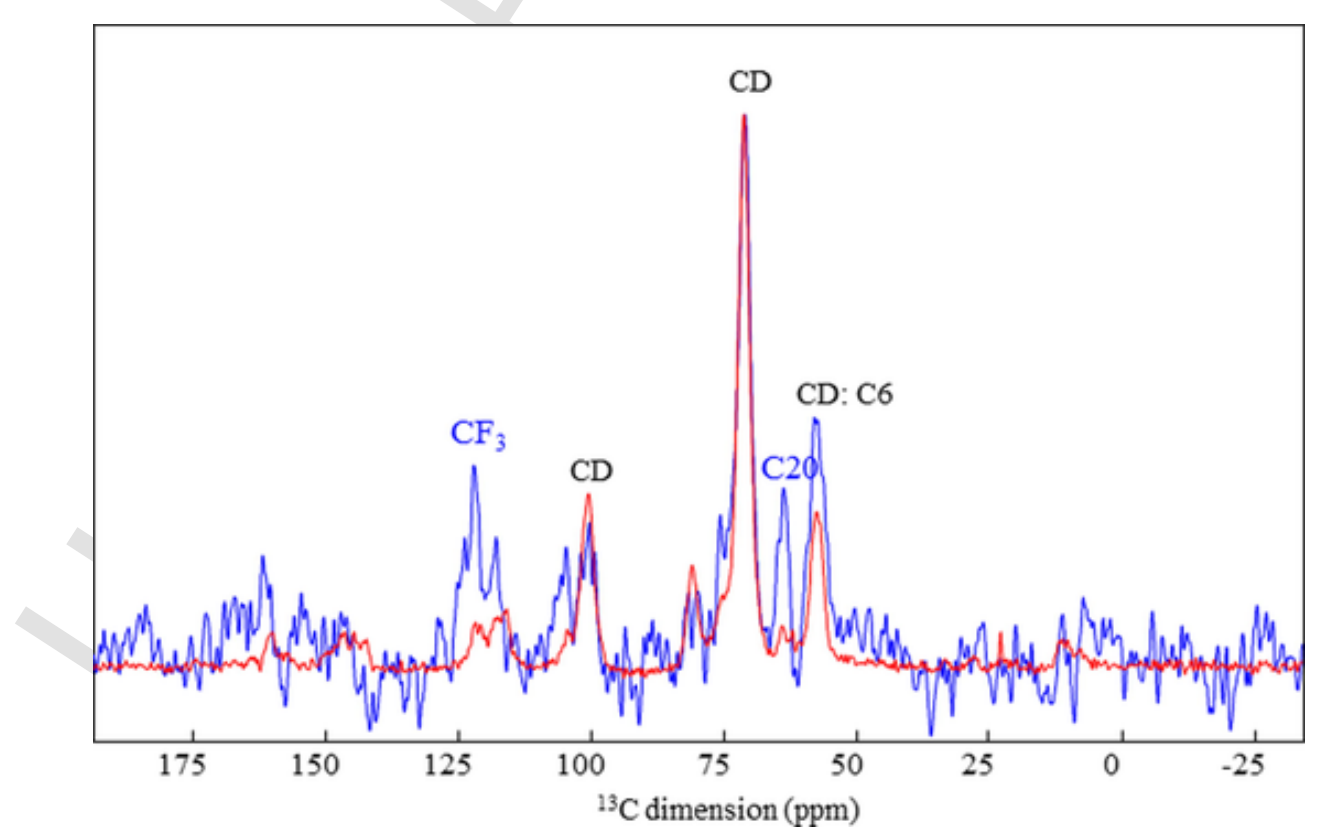

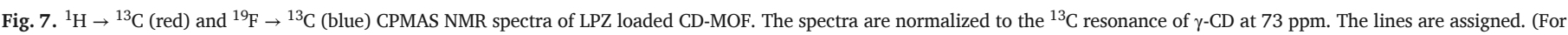
interpretation of the references to colour in this figure legend, the reader is referred to the web version of this article.) 


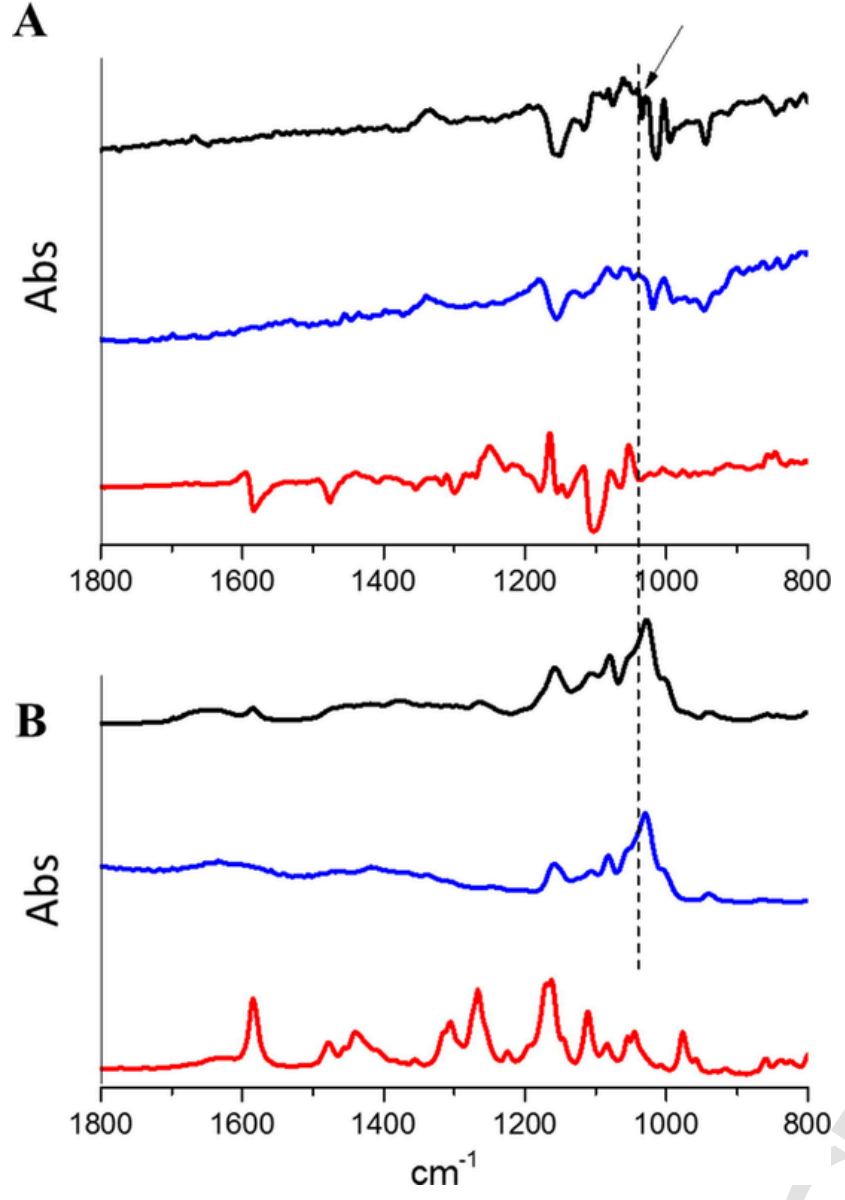

Fig. 8. (A) VCD spectrum of (R)-LPZ (red), bare CD-MOF (blue), and (R)-LPZ loaded CD-MOF (black), together with their absorption spectrum (B). The vertical scale of the VCD spectra is multiplied by $10^{4}$. (For interpretation of the references to colour in this figure legend, the reader is referred to the web version of this article.)

forms an inclusion complex was 40 times higher than for the CTAB:CD complex. This again supports the fact that during CD-MOF synthesis, $\mathrm{LPZ}$ molecules replace CTAB ones and only trace CTAB is found in the final LPZ-loaded CD-MOFs.

$\mathrm{K}=\mathrm{e}^{-\frac{\Delta G}{R T}}$

\subsection{Thermal stability of $L P Z$ as free or encapsulated form}

Once demonstrated that LPZ was embedded inside the $\gamma$-CDs of the CD-MOFs, it was interesting to study if this peculiar configuration allows improving its stability inside the dried particles stored at room temperature up to three years. To do so, thermal decomposition was investigated by TGA-FTIR analysis, combining the TGA ability to accurately and measure weight loss during heating and FTIR allowing to chemically identify the nature of the gas evolved from the sample decomposition.

TGA weight loss thermograms and the corresponding derivative curves (DTG) for LPZ, CD-MOF, and LPZ loaded CD-MOF crystals are shown in Fig. 10A and B. Free LPZ degraded in two steps, with specific temperatures of around $190{ }^{\circ} \mathrm{C}$ and $315{ }^{\circ} \mathrm{C}$, respectively. Total IR absorbance spectra were recorded when these degradation events occurred (Fig. S12). As illustrated in Fig. 10C, the FTIR spectra corresponding to the first gas departure at $190{ }^{\circ} \mathrm{C}$ corresponds well to 2,2,2-trifluoroethanol $\left(\mathrm{C}_{2} \mathrm{H}_{3} \mathrm{~F}_{3} \mathrm{O}\right)$. The FTIR spectra of the second departure at around $315{ }^{\circ} \mathrm{C}$ showed bands at around $1476 \mathrm{~cm}^{-1}$, which were assigned to the departure of LPZ aromatic rings (Fig. S13).
In contrast to LPZ, CD-MOF crystals exhibited different thermal degradation stages with two weight loss regions at around $55{ }^{\circ} \mathrm{C}$ and $300{ }^{\circ} \mathrm{C}$. First gas departure at around $55^{\circ} \mathrm{C}$ in CD-MOFs thermograms was attributed to isopropanol traces (Fig. S14), as this solvent was used to wash the particles. CD-MOF particles decomposed at around $300{ }^{\circ} \mathrm{C}$.

Finally, three decomposition steps were observed for LPZ-loaded CD-MOFs (Fig. 10, blue curves). The first departure corresponds to isopropanol traces, as in the case of empty CD-MOFs. The 2,2,2-trifluoroethanol vapor production corresponding to LPZ decomposition was identified in both second and third departures (around 200 and $310{ }^{\circ} \mathrm{C}$, respectively). Remarkably, the departure of 2,2,2-trifluoroethanol from LPZ-loaded CD-MOFs was dramatically delayed as compared to the pure LPZ $\left(190^{\circ} \mathrm{C} \& 310^{\circ} \mathrm{C}\right.$ for pure LPZ and LPZ-loaded CD-MOFs, respectively). This delay corresponds to an increased in the stability of the drug upon decomposition which could be attributed to its embedding inside the CD-MOF matrix. These results are in line with the protective effects of LPZ embedding in native CDs upon thermal decomposition of LPZ (Lu et al., 2012).

\section{Conclusion}

LPZ-loaded CD-MOFs were successfully synthesized displaying regular cubic morphology, uniform size of around $5 \mu \mathrm{m}$ and high LPZ payloads up to $23 \pm 2 \mathrm{wt} \%$, which corresponds to a molar ratio of $1: 1$ between LPZ and $\gamma$-CD in the CD-MOFs. The drug was uniformly distributed inside the particles as assesses by STEM-EDX in the whole particles and in thin slices of them. Solid NMR enabled deciphering drug location and configuration inside CD-MOFs, which was well corroborated by molecular simulations and VCD studies.

The drug LPZ was well incorporated inside the constitutive $\gamma$-CDs cavities of the CD-MOFs, filling practically all of them. LPZ competes for the $\gamma$-CDs with the CTAB surfactant used to modulate the size of the CD-MOFs and replaces it, leading to the formation of crystals devoid of CTAB, but maintaining their high LPZ payloads, which is an advantage for biomedical applications.

Remarkably, the CD-MOF matrix protected the drug upon degradation, even after three years storage at room temperature.

\section{Declaration of Competing Interest}

The authors declare that they have no known competing financial interests or personal relationships that could have appeared to influence the work reported in this paper.

\section{Acknowledgements}

This work was supported by the French National Research Agency (ANR-10-LABX-0035, ANR-14-CE08-0017 and EuronanoMedIII PCInano) and the National Science and Technology Major Projects for "Major New Drugs Innovation and Development" (2018ZX09721002-009). We thank Pierre Florien (CEMHTI Orléans) for help in the $15 \mathrm{~N}$-NMR experiments, financial support from the IR-RMN-THC Fr3050 CNRS, the MIMA2-MET platform (https://doi.org/10.15454/1. 5572348210007727E12) and the Ile-de-France DIM RESPORE. We are grateful to Dr. Borja Moreira-Alvarez, Dr José M. Costa Fernandez and Dr. Jorge R. Encinar (University of Oviedo, Spain) for ICP-MS experiments. MP thanks the Région Centre-Val de Loire for a $\mathrm{PhD}$ fellowship. We thank Dr. Mohamed Haouas (ILV, Versailles) for the acquisition of the ${ }^{13} \mathrm{C}$ liquid NMR spectrum.

\section{Appendix A. Supplementary data}

Supplementary data to this article can be found online at https://doi. org/10.1016/j.ijpharm.2020.119442. 
A
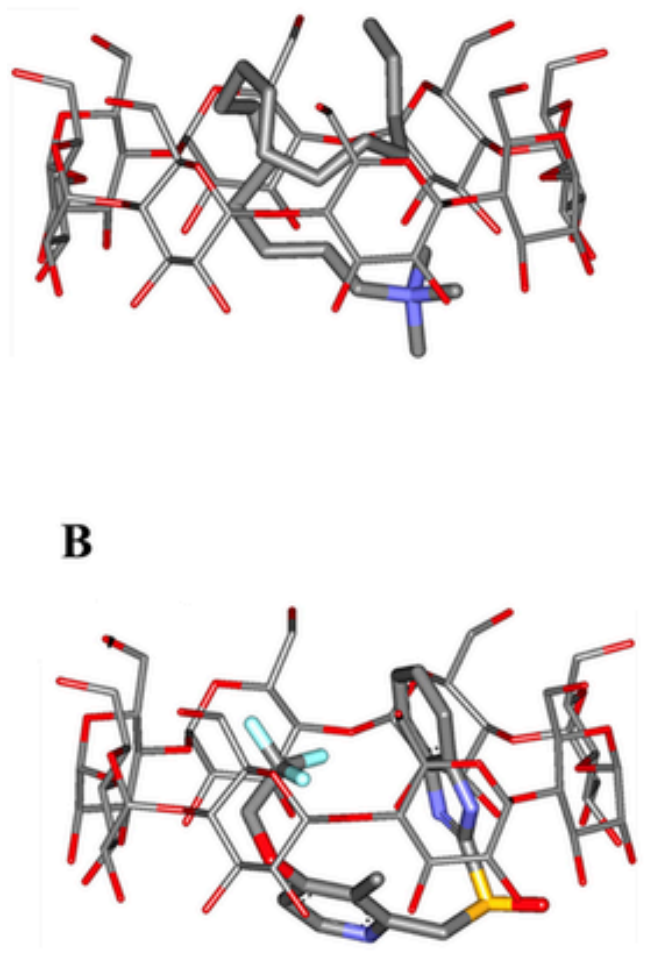

C

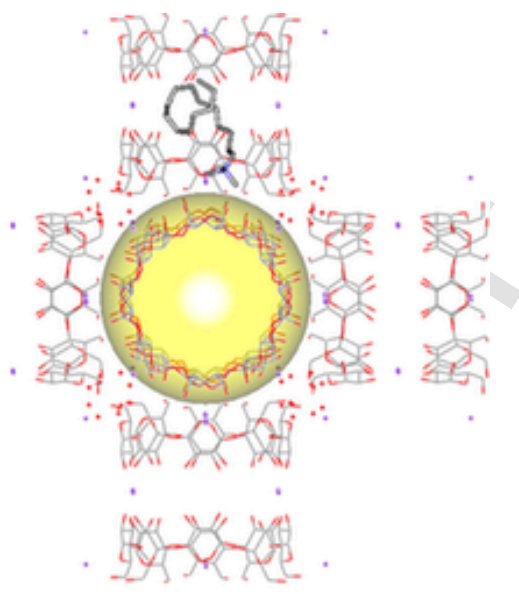

D

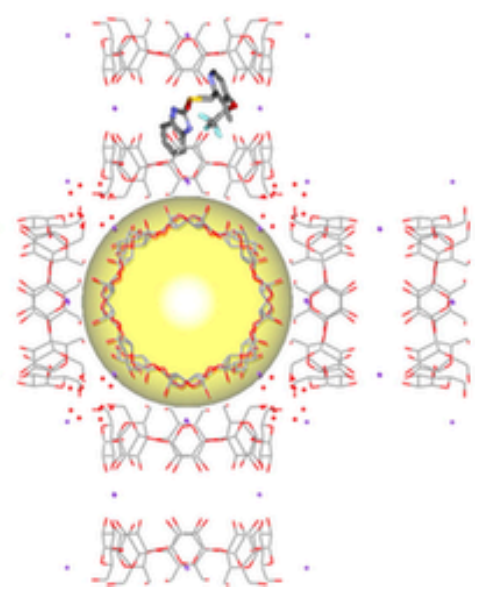

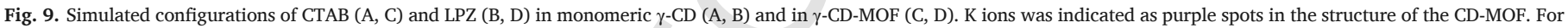

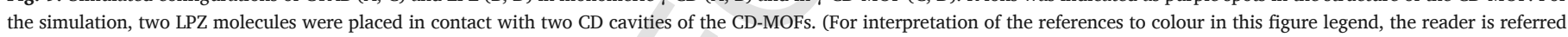
to the web version of this article.) 
$\mathbf{A}$

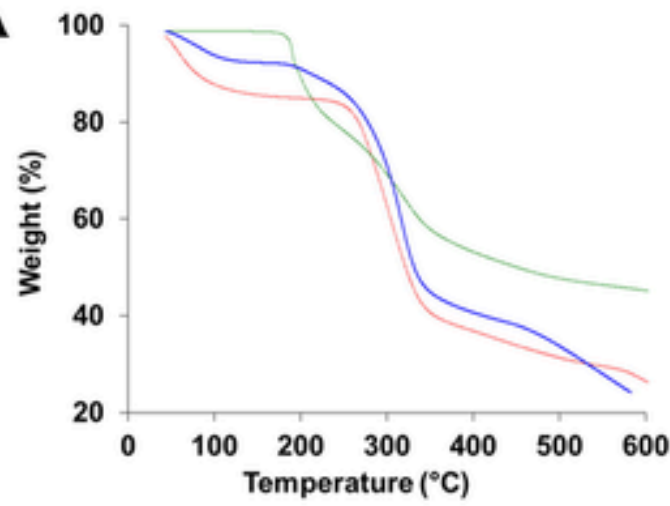

B

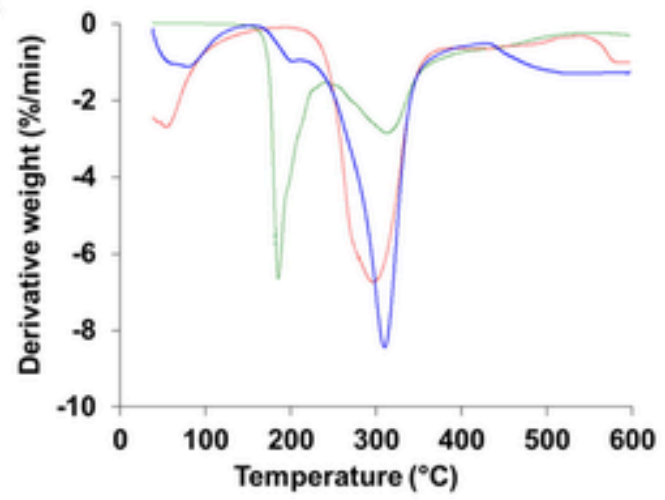

C

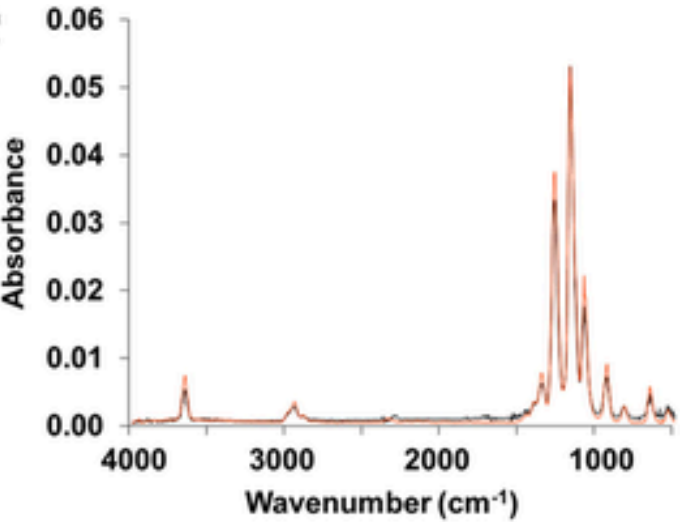

D

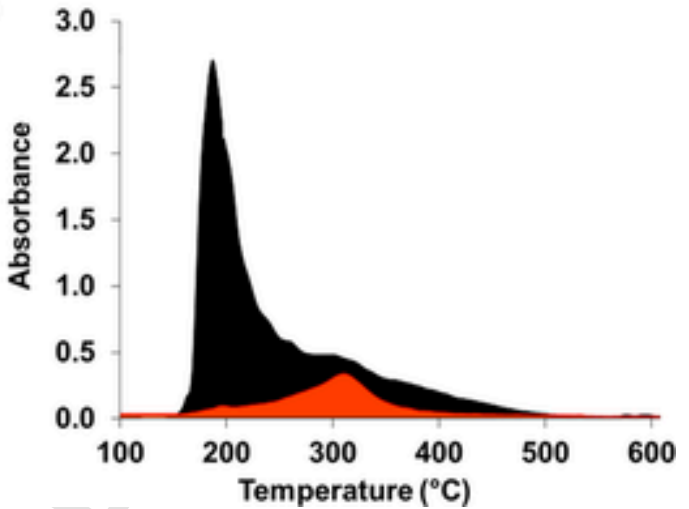

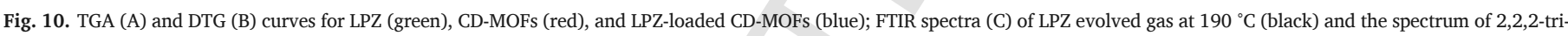

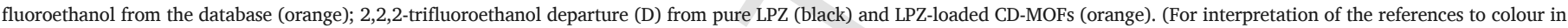
this figure legend, the reader is referred to the web version of this article.)

\section{References}

Abuçafy, M P, Caetano, B L, Chiari-Andréo, B G, Fonseca-Santos, B, do Santos, A M, Chorilli, M, Chiavacci, L A, 2018. Supramolecular cyclodextrin-based metal-organic frameworks as efficient carrier for anti-inflammatory drugs. Eur. J. Pharm. Biopharm. 127, 112-119.

Allouche, A, 2012. Software news and updates gabedit: a graphical user interface for computational chemistry softwares. J. Comput. Chem. 31, 174-182.

Arima, H, Hayashi, Y, Higashi, T, Motoyama, K, 2015. Recent advances in cyclodextrin delivery techniques. Expert Opin. 12, 1425-1441.

Battu, S, Pottabathini, V, 2015. Hydrolytic degradation study of lansoprazole, identification, isolation and characterisation of base degradation product. Am. J. Anal. Chem. 6, 145-155.

Bekiroglu, S, Kenne, L, Sandström, C, 2003. 1H NMR studies of maltose, maltoheptaose, $\alpha-, \beta-$, and $\gamma$-cyclodextrins, and complexes in aqueous solutions with hydroxy protons as structural probes. J. Org. Chem. 68, 1671-1678.

Buffeteau, T, Lagugné-Labarthet, F, Sourisseau, C, 2005. Vibrational circular dichroism in general anisotropic thin solid films: measurement and theoretical approach. Appl. Spectrosc. 59, 732-745.

Cheng, J G, Yu, H J, Chen, Y, Liu, Y, 2018. Selective binding and controlled release of anticancer drugs by polyanionic cyclodextrins. Bioorganic Med. Chem. 26, 2287-2290.

Declerck, V, Pérez-Mellor, A, Guillot, R, Aitken, D J, Mons, M, Zehnacker, A, 2019. Vibrational circular dichroism as a probe of solid-state organisation of derivatives of cyclic $\beta$-amino acids: cis- and trans-2-aminocyclobutane-1-carboxylic acid. Chirality $31,547-560$.

DellaGreca, M, Iesce, M R, Previtera, L, Rubino, M, Temussi, F, Brigante, M, 2006. Degradation of lansoprazole and omeprazole in the aquatic environment. Chemosphere 63, 1087-1093.

Di Nunzio, M R, Wang, Y L, Douhal, A, 2013. Spectroscopy and dynamics of topotecan anti-cancer drug comprisedwithin cyclodextrins. J. Photochem. Photobiol. A Chem. $266,12-21$.

DiGiacinto, J L, Olsen, K M, Bergman, K L, Hoie, E B, 2000. Stability of suspension formulations of lansoprazole and omeprazole stored in amber-colored plastic oral syringes. Ann. Pharmacother. 34, 600-605.

Ekpe, A, Jacobsen, T, 1999. Effect of various salts on the stability of lansoprazole, omeprazole, and pantoprazole as determined by high-performance liquid chromatography. Drug Dev. Ind. Pharm. 25, 1057-1065.
Ferreira, M J G, García, A, Leonardi, D, Salomon, C J, Lamas, M C, Nunes, T G, 2015. $13 \mathrm{C}$ and $15 \mathrm{~N}$ solid-state NMR studies on albendazole and cyclodextrin albendazole complexes. Carbohydr. Polym. 123, 130-135.

Forgan, R S, Smaldone, R A, Gassensmith, J J, Furukawa, H, Cordes, D B, Li, Q, Wilmer, C E, Botros, Y Y, Snurr, R Q, Slawin, A M Z, Stoddart, J F, 2012. Nanoporous carbohydrate metal-organic frameworks. J. Am. Chem. Soc. 134, 406-417.

Furukawa, Y, Ishiwata, T, Sugikawa, K, Kokado, K, Sada, K, 2012. Nano- and microsized cubic gel particles from cyclodextrin metal-organic frameworks. Angew. Chem. Int. Ed. 124, 10718-10721.

Gasteiger, J, Marsili, M, 1980. Iterative partial equalization of orbital electronegativity-a rapid access to atomic charges. Tetrahedron 36, 3219-3228.

Gupta, H P, Saini, K, Dhingra, P, Pandey, R, 2007. Study of acid catalyzed reactions of proton pump inhibitors at D.M.E. Port. Electrochim. Acta 26, 433-448.

Han, Y, Liu, W, Huang, J, Qiu, S, Zhong, H, Liu, D, 2018. Cyclodextrin-based metal-organic frameworks (CD-MOFs) in pharmaceutics and biomedicine. Pharmaceutics 10, 1-21.

Hassan, A, Quinn, C M, Struppe, J, Sergeyev, I V, Zhang, C, Guo, C, Runge, B, Theint, T, Dao, H H, Jaroniec, C P, Berbon, M, Lends, A, Habenstein, B, Loquet, A, Kuemmerle, R, Perrone, B, Gronenborn, A M, Polenova, T, 2020. Sensitivity boosts by the CPMAS CryoProbe for challenging biological assemblies. J. Magn. Reson. 311, 106680.

Johnson, R L, Schmidt-Rohr, K, 2014. Quantitative solid-state 13C NMR with signal enhancement by multiple cross polarization. J. Magn. Reson. 239, 44-49.

Kundu, M, Roy, M N, 2017. Preparation, interaction and spectroscopic characterization of inclusion complex of a cyclic oligosaccharide with an antidepressant drug. J. Incl. Phenom. Macrocycl. Chem. 89, 177-187.

Li, X, Guo, T, Lachmanski, L, Manoli, F, Menendez-Miranda, M, Manet, I, Guo, Z, Wu, L, Zhang, J, Gref, R, 2017. Cyclodextrin-based metal-organic frameworks particles as efficient carriers for lansoprazole: Study of morphology and chemical composition of individual particles. Int. J. Pharm. 531, 424-432.

Liu, B, He, Y, Han, L, Singh, V, Xu, X, Guo, T, Meng, F, Xu, X, York, P, Liu, Z, Zhang, J, 2017. Microwave-assisted rapid synthesis of $\gamma$-cyclodextrin metal-organic frameworks for size control and efficient drug loading. Cryst. Growth Des. 17, 1654-1660.

Liu, B, Li, H, Xu, X, Li, X, Lv, N, Singh, V, Stoddart, J F, York, P, Xu, X, Gref, R, Zhang, $\mathrm{J}, 2016$. Optimized synthesis and crystalline stability of $\gamma$-cyclodextrin metal-organic frameworks for drug adsorption. Int. J. Pharm. 514, 212-219.

Liu, K H, Kim, M J, Jung, W M, Kang, W, Cha, I J, Shin, J G, 2005. Lansoprazole enantiomer activates human liver microsomal cyp2C9. Pharmacology 33, 209-213.

Loftsson, T, Brewster, M E, 1996. Pharmaceutical applications of cyclodextrins. 1. Drug solubilization and stabilization. J. Pharm. Sci. 85, 1017-1025.

Loftsson, T, Duchêne, D, 2007. Cyclodextrins and their pharmaceutical applications. Int. J. Pharm. 329, 1-11. 
Lu, Y, Guo, T, Qi, J, Zhang, J, Wu, W, 2012. Enhanced dissolution and stability of lansoprazole by cyclodextrin inclusion complexation: preparation, characterization, and molecular modeling. AAPS PharmSciTech 13, 1222-1229.

Lv, N, Guo, T, Liu, B, Wang, C, Singh, V, Xu, X, Li, X, Chen, D, Gref, R, Zhang, J, 2016. Improvement in thermal stability of sucralose by $\gamma$-cyclodextrin metal-organic frameworks. Pharm. Res. 34, 269-278.

Martineau, C, Engelke, F, Taulelle, F, 2011. Multiple resonance heteronuclear decoupling under MAS: dramatic increase of spectral resolution at moderate magnetic field and MAS frequencies. J. Magn. Reson. 212, 311-319.

Massiot, D, Fayon, F, Capron, M, King, I, Le Calvé, S, Alonso, B, Durand, J O, Bujoli, B, Gan, Z, Hoatson, G, 2002. Modelling one- and two-dimensional solid-state NMR spectra. Magn. Reson. Chem. 40, 70-76.

Merten, C, Kowalik, T, Hartwig, A, 2008. Vibrational circular dichroism spectroscopy of solid polymer films: Effects of sample orientation. Appl. Spectrosc. 62, 901-905.

Michida, W, Ezaki, M, Sakuragi, M, Guan, G, Kusakabe, K, 2015. Crystal growth of cyclodextrin-based metal-organic framework with inclusion of ferulic acid. Cryst. Res. Technol. 50, 556-559.

Moussa, Z, Hmadeh, M, Abiad, M G, Dib, O H, Patra, D, 2016. Encapsulation of curcumin in cyclodextrin-metal organic frameworks: dissociation of loaded CD-MOFs enhances stability of curcumin. Food Chem. 212, 485-494.

Ni, Q Z, Yang, F, Can, T V, Sergeyev, I V, D’Addio, S M, Jawla, S K, Li, Y, Lipert, M P, $\mathrm{Xu}, \mathrm{W}$, Williamson, R T, Leone, A, Griffin, R G, Su, Y, 2017. In situ characterization of pharmaceutical formulations by dynamic nuclear polarization enhanced MAS NMR. J. Phys. Chem. B 121, 8132-8141.

Pasic, M., 2008. Study to design stable lansoprazole pellets (Ph.D. thesis). Univ. Basel.

Pérez-Mellor, A, Zehnacker, A, 2017. Vibrational circular dichroism of a 2,5-diketopiperazine (DKP) peptide: evidence for dimer formation in cyclo LL or LD diphenylalanine in the solid state. Chirality 29, 89-96.

Qiu, C, Wang, J, Qin, Y, Fan, H, Xu, X, Jin, Z, 2018. Green synthesis of cyclodextrin-based metal-organic frameworks through the seed-mediated method for the encapsulation of hydrophobic molecules. J. Agric. Food Chem. 66, 4244-4250.

Qiu, J., Li, X., Gref, R., Vargas-Berenguel, A., 2020. Carbohydrates in metal organic frameworks: Supramolecular assembly and surface modification for biomedical applications, in: Mozafari, M. (Ed.), Metal Organic Frameworks for Biomedical Applications. pp. $445-465$

Rajendiran, N, Sankaranarayanan, R K, Saravanan, J, 2014. A study of supramolecular host-guest interaction of dothiepin and doxepin drugs with cyclodextrin macrocycles. J. Mol. Struct. 1067, 252-260.
Rajkumar, T, Kukkar, D, Kim, K H, Sohn, J R, Deep, A, 2019. Cyclodextrin-metal-organic framework (CD-MOF): From synthesis to applications. J. Ind. Eng. Chem. 72, 50-66.

Ramulu, K, Rao, B M, Someswara Rao, N, 2013. Identification, isolation and characterization of potential degradation product in idebenone drug substance. Rasayan J. Chem. 6, 324-333.

Shankar, G, Borkar, R M, Suresh, U, Guntuku, L, Naidu, V G M, Nagesh, N, Srinivas, R, 2017. Forced degradation studies of lansoprazole using LC-ESI HRMS and 1H-NMR experiments: in vitro toxicity evaluation of major degradation products. J. Mass Spectrom. 52, 459-471.

Skorupska, E, Jeziorna, A, Kazmierski, S, Potrzebowski, M J, 2014. Recent progress in solid-state NMR studies of drugs confined within drug delivery systems. Solid State Nucl. Magn. Reson. 57-58, 2-16.

Smaldone, R A, Forgan, R S, Furukawa, H, Gassensmith, J J, Slawin, A M Z, Yaghi, O M, Stoddart, J F, 2010. Metal-organic frameworks from edible natural products. Angew. Chem. Int. Ed. 49, 8630-8634.

Upreti, M, Strassburger, K, Chen, Y L, Wu, S, Prakash, I, 2011. Solubility enhancement of steviol glycosides and characterization of their inclusion complexes with gamma-cyclodextrin. Int. J. Mol. Sci. 12, 7529-7553.

Vogt, F G, Strohmeier, M, 2012. 2D solid-state NMR analysis of inclusion in drug-cyclodextrin complexes. Mol. Pharm. 9, 3357-3374.

Wulff, M, Aldén, M, Tegenfeldt, J, 2002. Solid-state NMR investigation of indomethacin-cyclodextrin complexes in PEG 6000 carrier. Bioconjug. Chem. 13, 240-248.

Xu, J, Wu, L, Guo, T, Zhang, G, Wang, C, Li, H, Li, X, Singh, V, Chen, W, Gref, R, Zhang, J, 2019. A "Ship-in-a-Bottle" strategy to create folic acid nanoclusters inside the nanocages of $\gamma$-cyclodextrin metal-organic frameworks. Int. J. Pharm. 556, 89-96.

Ye, Y, Sun, Y, Zhao, H, Lan, M, Gao, F, Song, C, Lou, K, Li, H, Wang, W, 2013. A nove lactoferrin-modified $\beta$-cyclodextrin nanocarrier for brain-targeting drug delivery. Int. J. Pharm. 458, 110-117.

Zhao, Yong, Sanner, Michel F, 2007. FLIPDock: docking flexible ligands into flexible receptors. proteins. Struct. Funct. Bioinfomatics 68, 726-737.

Yu, C, Bourrelly, S, Martineau, C, Saidi, F, Bloch, E, Lavrard, H, Taulelle, F, Horcajada, P, Serre, C, Llewellyn, P L, Magnier, E, Devic, T, 2015. Functionalization of Zr-based MOFs with alkyl and perfluoroalkyl groups: the effect on the water sorption behavior. Dalt. Trans. 44, 19687-19692. 